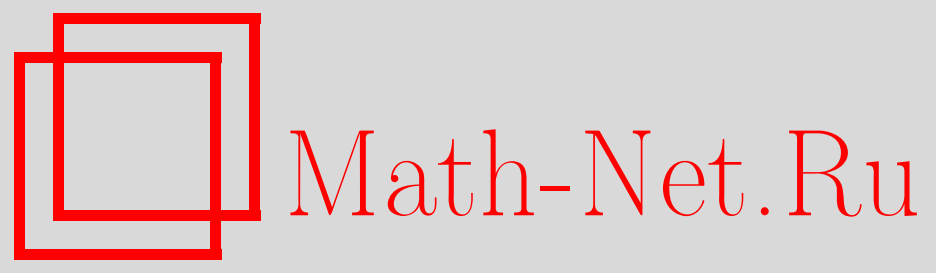

C. А. Назаров, Асимптотика собственных чисел на непрерывном спектре регулярно возмущенного квантового волновода, ТМФ, 2011, том 167, номер 2, 239-263

DOI: https://doi.org/10.4213/tmf6637

Использование Общероссийского математического портала Math-Net.Ru подразумевает, что вы прочитали и согласны с пользовательским соглашением http://www.mathnet.ru/rus/agreement

Параметры загрузки:

IP: 3.82 .47 .9

26 апреля 2023 г., 16:12:44

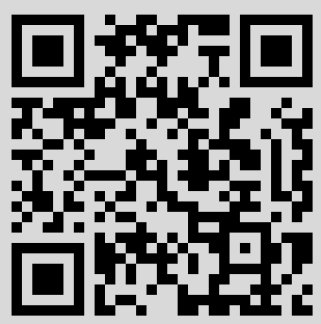




\title{
ФИЗИКА
}

Том 167, № 2

май, 2011

(C) 2011 г.

\author{
С. А. Назаров*
}

\section{АСИМПТОТИКА СОБСТВЕННЫХ ЧИСЕЛ НА НЕПРЕРЫВНОМ СПЕКТРЕ РЕГУЛЯРНО ВОЗМУЩЕННОГО КВАНТОВОГО ВОЛНОВОДА}

\begin{abstract}
Установлено, что путем подбора гладкого локального возмущения границы плоского квантового волновода можно образовать собственное число около любого наперед заданного порога непрерывного спектра и соответствующую захваченную волну, экспоненциально затухающую на бесконечности. Дано асимптотическое истолкование аномалий Вуда - быстрого изменения дифракционной картины при вариации околопороговой частоты волны. Исследование основано на анализе вспомогательного объекта - унитарной расширенной матрицы рассеяния.
\end{abstract}

Ключевые слова: квантовый волновод, регулярное возмущение границы, асимптотика собственного числа на непрерывном спектре, аномалии Вуда.

\section{1. ПОСТАНОВКА ЗАДАЧИ}

Пусть $\Pi^{0}=\mathbb{R} \times(0,1)$ - единичная полоса и $\Pi^{\varepsilon}$ - полоса с регулярным пологим возмущением одной из сторон,

$$
\Pi^{\varepsilon}=\left\{x=\left(x_{1}, x_{2}\right): x_{1} \in \mathbb{R}=(-\infty,+\infty),-\varepsilon H\left(x_{1}\right)<x_{2}<1\right\},
$$

где $\varepsilon>0$ - малый безразмерный параметр, $H$ - гладкая функция с носителем на сегменте $[-l, l], l>0$. Интерпретируя множество (1) как квантовый волновод, рассмотрим спектральную задачу Дирихле

$$
-\Delta u^{\varepsilon}(x)=\lambda^{\varepsilon} u^{\varepsilon}(x), \quad x \in \Pi^{\varepsilon}, \quad u^{\varepsilon}(x)=0, \quad x \in \partial \Pi^{\varepsilon},
$$

где $\Delta$ - оператор Лапласа, $\lambda^{\varepsilon}$ - спектральный параметр. Известно (см., например, $[1])$, что непрерывный спектр задачи (2) занимает луч $\left[\pi^{2},+\infty\right)$, который порогами $\pi^{2} n^{2}, n \in \mathbb{N}=\{1,2,3, \ldots\}$, разбит на интервалы постоянной кратности. Именно, если

$$
\lambda^{\varepsilon} \in\left(\pi^{2}(n-1)^{2}, \pi^{2} n^{2}\right),
$$

* Институт проблем машиноведения РАН, Санкт-Петербург, Россия.

E-mail: srgnazarov@yahoo.co.uk 
то в прямом волноводе $\Pi^{0}$ возникает $2(n-1)$ осциллирующих волн

$$
w_{k}^{ \pm}(x)=\left(\lambda^{\varepsilon}-\pi^{2} k^{2}\right)^{-1 / 4} \sin \left(\pi k x_{2}\right) e^{ \pm i x_{1} \sqrt{\lambda^{\varepsilon}-\pi^{2} k}}, \quad k=1, \ldots, n-1 .
$$

Цель статьи - убедиться в том, что путем подбора профильной функции $H$ в формуле (1) при малом $\varepsilon>0$ можно на любом интервале (3) с $n>1$ образовать собственное число

$$
\lambda_{n}^{\varepsilon}=\pi^{2} n^{2}-\varepsilon^{2} \mu_{n}+O\left(\varepsilon^{3}\right), \quad \mu_{n}>0,
$$

задачи (2). Для упрощения построений и изложения предполагаем, что $H$ - четная функция и $H(0)=0$ (по поводу снятия этих ограничений см. обсуждение в разделе 9), и сужаем задачу (2) на половину $\Pi_{+}^{\varepsilon}=\left\{x \in \Pi^{\varepsilon}: x_{1}>0\right\}$ волновода (1), задав на сечении $\varpi_{0}^{\varepsilon}=\left\{x \in \Pi^{\varepsilon}: x_{1}=0\right\}$ искусственное краевое условие Неймана, т. е. рассматриваем смешанную краевую задачу

$$
\begin{gathered}
-\Delta u^{\varepsilon}(x)=\lambda^{\varepsilon} u(x), \quad x \in \Pi_{+}^{\varepsilon}, \quad-\partial_{1} u^{\varepsilon}\left(0, x_{2}\right)=0, \quad 0<x_{2}<1, \\
u^{\varepsilon}\left(x_{1}, 1\right)=0, u^{\varepsilon}\left(x_{1}, \varepsilon H\left(x_{1}\right)\right)=0, \quad x_{1}>0 .
\end{gathered}
$$

С другой стороны, собственная функция $u_{+}^{\varepsilon}$, отвечающая собственному числу $\lambda^{\varepsilon}$ задачи (6), (7) и принадлежащая пространству Соболева $H^{2}\left(\Pi_{+}^{\varepsilon}\right)$, после четного продолжения с $\Pi_{+}^{\varepsilon}$ на $\Pi^{\varepsilon}$ порождает спектральную пару $\left\{u^{\varepsilon}, \lambda^{\varepsilon}\right\}$ задачи (2). Собственная функция $u^{\varepsilon} \in H^{2}\left(\Pi^{\varepsilon}\right)$ экспоненциально затухает на бесконечности и потому называется захваченной волной или локализованным решением.

В разделе 7 будет показано, что постановка на торце $\varpi_{0}^{\varepsilon}$ искусственных условий Дирихле не приводит к успеху, поскольку задача Дирихле в $\Pi_{+}^{\varepsilon}$ не имеет собственных чисел при малом $\varepsilon>0$.

Собственное число $\lambda^{\varepsilon} \in\left(0, \pi^{2}\right)$ принадлежит дискретному спектру задачи (6), (7), но собственное число $\lambda^{\varepsilon} \geqslant \pi^{2}$ содержится в непрерывном спектре, т. е. попадает только в точечный спектр. Поиску и изучению асимптотик собственных чисел ниже порога $\lambda_{\dagger}=\pi^{2}$ непрерывного спектра посвящено большое количество публикаций. В работах [2]-[5] установлено существование и построена асимптотика собственного числа $\lambda^{\varepsilon}<\lambda_{\dagger}$ для слабо изогнутых волноводов. В статьях [6]-[11] исследованы плоские и многомерные волноводы с регулярными и сингулярными локальными возмущениями границы, найдены разнообразные условия существования и отсутствия дискретного спектра, а также указаны асимптотики.

Дискретный спектр рассматриваемой задачи (2) в волноводе (1) изучен в работе [6], где проверено, что при условии

$$
\eta_{0}=\pi^{2} \int_{\mathbb{R}} H\left(x_{1}\right) d x_{1}<0,
$$

означающем, что возмущение волновода привело к уменьшению его объема, интервал $\left(0, \pi^{2}\right)$ свободен от спектра задачи, но в случае $\eta_{0}>0$ (приращение объема положительно) содержит единственную точку

$$
\lambda^{\varepsilon}=\pi^{2}-\varepsilon^{2} \eta_{0}^{2}+O\left(\varepsilon^{3}\right) .
$$


Кроме того, в работе [7] показано, что в критической ситуации $\eta_{0}=0$ собственное число задачи (2) может как отсутствовать, так и появиться на интервале $\left(0, \pi^{2}\right)$ в зависимости от профильной функции $H$ из формулы (1).

Результатов о существовании и асимптотике собственных чисел, вкрапленных в непрерывный спектр, несоизмеримо меньше. Отметим статью [10], в которой сформулированы условия отсутствия собственных чисел в $\left(c_{n} \varepsilon\right)$-окрестности точки $\pi^{2} n^{2}$, а также метод постановки искусственных краевых условий Дирихле на линии (плоскости) поперечной симметрии волновода, приводящий к обнаружению собственных чисел [12]. Понятно, что у волновода (1) такой симметрии нет, и поэтому подробного описания этого метода приводить не будем. Укажем лишь, что согласно работе [13] нарушение поперечной симметрии волновода с препятствием (т. е. сдвиг последнего) приводит к исчезновению собственного числа из непрерывного спектра и образованию точки комплексного резонанса.

В настоящей работе развивается подход к построению асимптотики собственных чисел, намеченный в статьях [14], [15] и основанный на анализе вспомогательного объекта - расширенной матрицы рассеяния, которая была введена в работах [16] и [17] для конических и цилиндрических областей соответственно. Собственные числа блоков этой унитарной матрицы (см. теорему 2) служат идентификатором захваченных волн.

Полученный результат, касающийся асимптотики собственного числа $\lambda^{\varepsilon}>\pi^{2}$, даже по формулировке существенно отличается от привычного (9). Введем необходимые обозначения и требования к профильной функции $H$, которую следует представить в виде

$$
H\left(x_{1}\right)=H_{0}\left(x_{1}\right)+\tau_{1} H_{1}\left(x_{1}\right)+\cdots+\tau_{n-1} H_{n-1}\left(x_{1}\right),
$$

где $\tau_{1}, \ldots, \tau_{n-1}-$ дополнительные малые параметры. Для основной составляющей $H_{0}$ предполагается, что выполнены соотношения

$$
\begin{gathered}
\eta_{n, 0}=\eta_{n}\left(H_{0}\right):=2 \pi^{2} n^{2} \int_{0}^{l} H_{0}\left(x_{1}\right) d x_{1}>0 \\
\eta_{k, 0}=\eta_{k}\left(H_{0}\right):=\frac{2 \pi n k}{\sqrt{n^{2}-k^{2}}} \int_{0}^{l} H_{0}\left(x_{1}\right) \cos \left(x_{1} \pi \sqrt{n^{2}-k^{2}}\right) d x_{1}=0
\end{gathered}
$$

(ср. формулы (11) и (8)). "Поправочные" слагаемые в (10) подчинены следующим условиям "ортогональности" и "нормировки":

$$
\eta_{k}\left(H_{j}\right)=\delta_{k, j}, \quad k, j=1, \ldots, n-1,
$$

где $\delta_{k, j}$ - символ Кронекера. Приведем основной результат работы.

Теорема 1. Зафиксируем натуральное число $n>1$. При выполнении требований (10)-(13) найдутся такие $\varepsilon_{0}>0$ и $K>0$, что при $\varepsilon \in\left(0, \varepsilon_{0}\right]$ существуют параметры

$$
\tau_{j} \in(-K \varepsilon, K \varepsilon), \quad j=1, \ldots, n-1,
$$

при которых у задачи (2) в волноводе $\Pi^{\varepsilon}$, заданном формулами (1) и (10), на интервале $\left(\pi^{2}(n-1)^{2}, \pi^{2} n^{2}\right)$ имеется единственное собственное число $\lambda^{\varepsilon}$, причем

$$
\left|\lambda^{\varepsilon}-\pi^{2} n^{2}+\eta_{n, 0}^{2} \varepsilon^{2}\right| \leqslant K \varepsilon^{3} .
$$


Статья разбита на разделы так, чтобы отделить формальные, в значительной степени простые, вычисления от их строгих доказательств, зачастую громоздких и технически сложных. В разделе 2 дано определение расширенной матрицы рассеяния, а также описаны ее свойства и приведено достаточное условие захвата волны [17]. В разделе 3 на основе техники весовых пространств с отделенной асимптотикой (см. обзор [18]) изучен оператор задачи (6), (7) и, в частности, показано, что достаточное условие оказывается и необходимым для рассматриваемой конкретной задачи. Асимптотики элементов расширенной матрицы рассеяния построены в разделе 4 и обоснованы в разделе 5 , а в разделе 6 доказано существование собственного числа (5). Единственность проверена в разделе 7. Асимптотическое истолкование аномалий Вуда, связанных с быстрой изменяемостью дифракционной картины около порогов, дано в разделе 8. В разделе 9 обсуждаются возможные обобщения и приведены пояснения к теореме 8 о существовании собственного числа задачи (2) без дополнительных требований к профильной функции $H$ в формуле (1).

\section{2. ВОЛНЫ И РАСШИРЕННАЯ МАТРИЦА РАССЕЯНИЯ}

В соответствии со знаком волнового числа $\pm\left(\lambda^{\varepsilon}-\pi^{2} k^{2}\right)^{1 / 2}$ волна $w_{k}^{+}$называется уходящей, а волна $w_{k}^{-}-$приходящей. Условия излучения Зоммерфельда допускают в асимптотическом разложении на бесконечности решения неоднородной задачи $(6),(7)$ только уходящие волны и обеспечивают существование следующих решений однородной задачи:

$$
u_{k}^{\varepsilon}(x)=w_{k}^{-}(x)+\sum_{j=1}^{n-1} s_{j k}^{\varepsilon} w_{k}^{+}(x)+O\left(e^{-x_{1} \sqrt{\pi^{2} n^{2}-\lambda^{\varepsilon}}}\right), \quad k=1, \ldots, n-1 .
$$

Коэффициенты отражения $s_{j k}^{\varepsilon}$ образуют матрицу рассеяния $s^{\varepsilon}$ размера $(n-1) \times(n-$ $1)$, которая оказывается унитарной благодаря нормирующим множителям в (4).

В работе [17] предложены к рассмотрению линейные комбинации экспоненциальных волн

$$
w_{n}^{ \pm}(x)=\left(4\left(\pi^{2} n^{2}-\lambda^{\varepsilon}\right)\right)^{-1 / 4} \sin \left(\pi n x_{2}\right)\left(e^{x_{1} \sqrt{\pi^{2} n^{2}-\lambda^{\varepsilon}}} \mp i e^{-x_{1} \sqrt{\pi^{2} n^{2}-\lambda^{\varepsilon}}}\right) .
$$

Кроме того, из результатов работы [17], относящихся к общим формально самосопряженным эллиптическим краевым задачам (см. ниже замечание), вытекает существование следующих $n$ решений задачи (6), (7):

$$
U_{p}^{\varepsilon}(x)=w_{p}^{-}(x)+\sum_{q=1}^{n} S_{q p}^{\varepsilon} w_{q}^{+}(x)+O\left(e^{-x_{1} \sqrt{\pi^{2}(n+1)^{2}-\lambda^{\varepsilon}}}\right), \quad p=1, \ldots, n .
$$

Матрица $S^{\varepsilon}$ размером $n \times n$, составленная из коэффициентов $S_{q p}^{\varepsilon}$ разложений (18) названа в работах [16], [17] расширенной матрицей рассеяния. Определение, предложенное в работе [17], отличается от широко принятого понятия расширенной матрицы рассеяния ${ }^{1)}$ (ср. [13]) тремя моментами. Во-первых, из-за присутствия в правой части (18) экспоненциально растущих волн решения $u_{k}^{\varepsilon}$ и $U_{k}^{\varepsilon}$ с номерами

\footnotetext{
1) В английской терминологии есть различие: augmented и extended scattering matrices.
} 
$k=1, \ldots, n-1$, вообще говоря, не совпадают, т. е. матрица $s^{\varepsilon}$ не служит верхним левым $(n-1) \times(n-1)$-блоком матрицы

$$
S^{\varepsilon}=\left(\begin{array}{ll}
S_{\bullet \bullet}^{\varepsilon} & S_{\bullet n}^{\varepsilon} \\
S_{n \bullet}^{\varepsilon} & S_{n n}^{\varepsilon}
\end{array}\right)
$$

(ср. далее формулу (29)). Во-вторых, $S^{\varepsilon}$ - унитарная матрица, и это обстоятельство оказывается весьма важным во многих вопросах. Наконец, в-третьих, матрица (19) имеет конечные размеры, а ее бесконечномерный аналог - оператор фиктивного рассеяния [19] - в настоящей статье не используется.

Для того чтобы проверить свойство унитарности матрицы (19), подставим решения $U_{p}$ и $U_{q}$ в формулу Грина на усеченном волноводе $\Pi_{+}^{\varepsilon}(R)=\left\{x \in \Pi_{+}^{\varepsilon}: x_{1}<R\right\}$ и получим равенство

$$
0=Q_{R}\left(U_{p}^{\varepsilon}, U_{q}^{\varepsilon}\right)=\int_{0}^{1}\left(\overline{U_{q}^{\varepsilon}\left(R, x_{2}\right)} \partial_{1} U_{p}^{\varepsilon}\left(R, x_{2}\right)-U_{p}^{\varepsilon}\left(R, x_{2}\right) \overline{\partial_{1} U_{q}^{\varepsilon}\left(R, x_{2}\right)}\right) d x_{2} .
$$

Непосредственные вычисления показывают, что благодаря введению нормирующих множителей во́лны (4) и (17) подчинены соотношениям

$$
Q_{R}\left(w_{j}^{ \pm}, w_{k}^{ \pm}\right)= \pm i \delta_{j, k}, \quad Q_{R}\left(w_{j}^{ \pm}, w_{k}^{\mp}\right)=0, \quad j, k=1, \ldots, n .
$$

Предельный переход $R \rightarrow+\infty$ устраняет из интеграла (20) экспоненциально малые остатки, и поэтому

$$
0=\lim _{R \rightarrow+\infty} Q_{R}\left(w_{p}^{-}+\sum_{j=1}^{n} S_{j p}^{\varepsilon} w_{j}^{+}, w_{q}^{-}+\sum_{k=1}^{n} S_{k q}^{\varepsilon} w_{k}^{+}\right)=-i \delta_{p, q}+i \sum_{j=1}^{n} \overline{S_{j q}^{\varepsilon}} S_{j p}^{\varepsilon} .
$$

Таким образом, $\left(S^{\varepsilon}\right)^{*} S^{\varepsilon}=\mathbb{I}_{n}$ - единичная $(n \times n)$-матрица, т. е. сопряженная $\left(S^{\varepsilon}\right)^{*}$ и обратная $\left(S^{\varepsilon}\right)^{-1}$ матрицы совпадают, а значит, $S^{\varepsilon}$ - унитарная матрица.

Достаточное условие [17] существования захваченной волны, привлекающее расширенную матрицу рассеяния, в рассматриваемом случае проверяется просто.

Теорема 2. Если $S_{n n}^{\varepsilon}=-1$, то решение $U_{n}^{\varepsilon}$ представляет собой захваченную волну, т.е. принадлежит пространству Соболева $H^{1}\left(\Pi_{+}^{\varepsilon}\right)$.

ДокАЗАТЕЛЬство. Поскольку $\left|S_{\bullet n}^{\varepsilon}\right|^{2}+\left|S_{n n}^{\varepsilon}\right|^{2}=1$ для столбца унитарной матрицы (19), видим, что $S_{\bullet}^{\varepsilon}=0$, следовательно, формула (18) для $U_{n}^{\varepsilon}$ выглядит так:

$$
U_{n}^{\varepsilon}(x)=w_{n}^{-}(x)-w_{n}^{+}(x)+O\left(e^{-x_{1} \sqrt{\pi^{2}(n+1)^{2}-\lambda^{\varepsilon}}}\right) .
$$

Осталось заметить, что $w_{n}^{-}(x)-w_{n}^{+}(x)=O\left(e^{-x_{1} \sqrt{\pi^{2} n^{2}-\lambda^{\varepsilon}}}\right)$ в силу определения (17).

Установим связь матриц $s^{\varepsilon}$ и $S^{\varepsilon}$, которая понадобится в разделе 8 .

Применим матричную форму записи: введем строку решений $U^{\varepsilon}=\left(U_{\bullet}^{\varepsilon}, U_{n}^{\varepsilon}\right)=$ $\left(U_{1}^{\varepsilon}, \ldots, U_{n-1}^{\varepsilon}, U_{n}^{\varepsilon}\right)$ и аналогичные строки волн $w^{ \pm}$. Формулы (18) теперь могут быть записаны кратко как

$$
U^{\varepsilon}=w^{-}+w^{+} S^{\varepsilon}+\cdots,
$$

здесь и далее многоточие заменяет экспоненциально затухающие слагаемые. 
Связь $w^{+}=\overline{w^{-}}$, вытекающая из определений (4) и (17), обеспечивает симметричность матрицы $S^{\varepsilon}$, т. е. равенство $\left(S^{\varepsilon}\right)^{\top}=S^{\varepsilon}$, где Т - знак транспонирования (без комплексного сопряжения). В самом деле, имеем

$$
\overline{U^{\varepsilon}}-1=\left(\overline{w^{-}}+\overline{w^{+}} \overline{S^{\varepsilon}}\right)\left(\overline{S^{\varepsilon}}\right)^{-1}+\cdots=w^{-}+w^{+}\left(\overline{S^{\varepsilon}}\right)^{-1}+\cdots,
$$

и, следовательно, в разности $R^{\varepsilon}=U^{\varepsilon}-\overline{U^{\varepsilon}}\left(\overline{S^{\varepsilon}}\right)^{-1}$ отсутствуют приходящие волны. Теперь аналогичное (20), (22) вычисление

$$
\begin{aligned}
0=\lim _{R \rightarrow+\infty} Q_{R}\left(R^{\varepsilon}, R^{\varepsilon}\right) & =\lim _{R \rightarrow+\infty} Q_{R}\left(w^{+}\left(S^{\varepsilon}-\left(\overline{S^{\varepsilon}}\right)^{-1}\right), w^{+}\left(S^{\varepsilon}-\left(\overline{S^{\varepsilon}}\right)^{-1}\right)\right)= \\
& =\left(S^{\varepsilon}-\left(\overline{S^{\varepsilon}}\right)^{-1}\right)^{*}\left(S^{\varepsilon}-\left(\overline{S^{\varepsilon}}\right)^{-1}\right)
\end{aligned}
$$

показывает, что $S^{\varepsilon}=\left(\overline{S^{\varepsilon}}\right)^{-1}$, и при учете унитарности матрицы $S^{\varepsilon}$ приходим к нужному соотношению

$$
S^{\varepsilon}=\left(\overline{S^{\varepsilon}}\right)^{-1}=\overline{\left(S^{\varepsilon}\right)^{-1}}=\overline{(S \varepsilon)^{*}}=\left(S^{\varepsilon}\right)^{\top} .
$$

Перепишем формулу (23) следующим образом:

$$
U_{\bullet}^{\varepsilon}=w_{\bullet}^{-}+w_{\bullet}^{+} S_{\bullet \bullet}^{\varepsilon}+w_{n}^{+} S_{n \bullet}^{\varepsilon}+\cdots, \quad U_{n}^{\varepsilon}=w_{n}^{-}+w_{\bullet}^{+} S_{\bullet n}^{\varepsilon}+w_{n}^{+} S_{n n}^{\varepsilon}+\cdots .
$$

Если $S_{n n}^{\varepsilon}=-1$, то, как проверено при доказательстве теоремы $2, S_{\bullet n}^{\varepsilon}=0$ и $S_{n \bullet}^{\varepsilon}=0$ согласно соотношению (24). В этом случае первая формула в (25) отличается от решений (16)

$$
u_{\bullet}^{\varepsilon}=\left(u_{1}^{\varepsilon}, \ldots, u_{n-1}^{\varepsilon}\right)=w_{\bullet}^{-}+w_{\bullet}^{+} s^{\varepsilon}+\ldots
$$

разве лишь линейной комбинацией захваченных волн. В результате $s^{\varepsilon}=S_{\bullet \bullet}^{\varepsilon}$ при условии $S_{n n}^{\varepsilon}=-1$.

Пусть теперь $S_{n n}^{\varepsilon} \neq-1$. Понимая под многоточием бесконечно малую величину порядка $O\left(e^{-x_{1} \sqrt{\pi^{2} n^{2}-\lambda^{\varepsilon}}}\right)$, отбрасываем из $(17)$ затухающую экспоненциальную волну и заменяем в $(25)$ волны $w_{n}^{ \pm}$их общей растущей составляющей

$$
w_{n}(x)=\left(4\left(\pi^{2} n^{2}-\lambda^{2}\right)\right)^{-1 / 4} \sin \left(\pi n x_{2}\right) e^{+x_{2} \sqrt{\pi^{2} n^{2}-\lambda^{\varepsilon}}} .
$$

Умножим вторую формулу в $(25)$ на $\left(S_{n n}^{\varepsilon}+1\right)^{-1} S_{n}^{\varepsilon}$ и вычтем из первой. В результате получаем, что

$$
\begin{aligned}
U_{\bullet}^{\varepsilon}-U_{n}^{\varepsilon}\left(S_{n n}^{\varepsilon}+1\right)^{-1} S_{n \bullet}^{\varepsilon}= & w_{\bullet}^{-}+w_{\bullet}^{+}\left(S_{\bullet \bullet}^{\varepsilon}-S_{\bullet n}^{\varepsilon}\left(S_{n n}^{\varepsilon}+1\right)^{-1} S_{n \bullet}^{\varepsilon}\right)+ \\
& +w_{n}\left(S_{n \bullet}^{\varepsilon}-\left(S_{n n}^{\varepsilon}+1\right)\left(S_{n n}^{\varepsilon}+1\right)^{-1} S_{n \bullet}^{\varepsilon}\right)+\cdots .
\end{aligned}
$$

Множитель при $w_{n}$ равен нулю, т. е. формулы (26) и (28) различаются лишь линейной комбинацией захваченных волн. Это наблюдение приводит к следующему утверждению.

Теорема 3. Справедлива формула

$$
s^{\varepsilon}=S_{\bullet \bullet}^{\varepsilon}-S_{\bullet n}^{\varepsilon}\left(S_{n n}^{\varepsilon}+1\right)^{-1} S_{n \bullet}^{\varepsilon},
$$

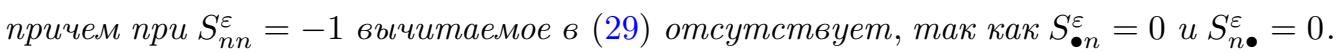




\section{3. ОПЕРАТОР ЗАДАЧИ В ВЕСОВЫХ КЛАССАХ}

Следуя [20] (см. также § 3.1 в [21]), обозначим через $W_{\beta}^{1}\left(\Pi_{+}^{\varepsilon}\right)$ весовое пространство Соболева, которое состоит из функций $u^{\varepsilon} \in H_{\mathrm{loc}}^{1}\left(\overline{\bar{\Pi}_{+}^{\varepsilon}}\right)$, обладающих конечной нормой

$$
\left\|u^{\varepsilon} ; W_{\beta}^{1}\left(\Pi_{+}^{\varepsilon}\right)\right\|=\left(\left\|e^{\beta x_{1}} \nabla u^{\varepsilon} ; L_{2}\left(\Pi_{+}^{\varepsilon}\right)\right\|^{2}+\left\|e^{\beta x_{1}} u^{\varepsilon} ; L_{2}\left(\Pi_{+}^{\varepsilon}\right)\right\|^{2}\right)^{1 / 2},
$$

где $\nabla$ - градиент. Понятно, что $W_{0}^{1}\left(\Pi_{+}^{\varepsilon}\right)$ - обычное пространство Соболева $H^{1}\left(\Pi_{+}^{\varepsilon}\right)$, но при $\beta>0$ в $W_{\beta}^{1}\left(\Pi_{+}^{\varepsilon}\right)$ содержатся только экспоненциально затухающие функции, а при $\beta<0$ - и затухающие, и растущие. Сопряженное пространство $W_{\beta}^{1}\left(\Pi_{+}^{\varepsilon}\right)^{*}$ можно отождествить с $W_{\beta}^{1}\left(\Pi_{+}^{\varepsilon}\right)$ путем расширения какого-либо скалярного произведения в $H^{1}\left(\Pi_{+}^{\varepsilon}\right)$. Под $W_{\beta}^{1,0}\left(\Pi_{+}^{\varepsilon}\right)$ мы подразумеваем подпространство функций из $W_{\beta}^{1}\left(\Pi_{+}^{\varepsilon}\right)$, удовлетворяющих условиям Дирихле (7).

Пусть $f^{\varepsilon} \in W_{-\beta}^{1,0}\left(\Pi_{+}^{\varepsilon}\right)^{*}-$ линейный непрерывный функционал на пространстве $W_{-\beta}^{1,0}\left(\Pi_{+}^{\varepsilon}\right)$. Под обобщенным решением неоднородной (с "правой частью" $f^{\varepsilon}$ ) задачи $(6),(7)$ в весовом классе $W_{\beta}^{1,0}\left(\Pi_{+}^{\varepsilon}\right)$ понимаем его элемент $u^{\varepsilon}$, удовлетворяющий интегральному тождеству [22] (краевой задаче в обобщенной постановке)

$$
\left(\nabla u^{\varepsilon}, \nabla v^{\varepsilon}\right)_{\Pi_{+}^{\varepsilon}}-\lambda^{\varepsilon}\left(u^{\varepsilon}, v^{\varepsilon}\right)_{\Pi_{+}^{\varepsilon}}=f^{\varepsilon}\left(v^{\varepsilon}\right), \quad v^{\varepsilon} \in W_{-\beta}^{1,0}\left(\Pi_{+}^{\varepsilon}\right),
$$

где $(\cdot, \cdot)_{\Pi_{+}^{\varepsilon}}-$ продолжение скалярного произведения в пространстве Лебега $L_{2}\left(\Pi_{+}^{\varepsilon}\right)$. Задаче (30) ставится в соответствие непрерывное отображение

$$
W_{\beta}^{1,0}\left(\Pi_{+}^{\varepsilon}\right) \ni u^{\varepsilon} \mapsto A_{\beta}^{\varepsilon}\left(\lambda^{\varepsilon}\right) u^{\varepsilon}=f^{\varepsilon} \in W_{-\beta}^{1,0}\left(\Pi_{+}^{\varepsilon}\right)^{*} .
$$

Ясно, что $A_{\beta}^{\varepsilon}\left(\lambda^{\varepsilon}\right)$ и $A_{-\beta}^{\varepsilon}\left(\lambda^{\varepsilon}\right)$ - взаимно сопряженные операторы.

Свойства отображения (31) зависят от чисел $\lambda^{\varepsilon}$ и $\beta$. Далее считаем, что

$$
\lambda^{\varepsilon} \in\left(\pi^{2}(n-1)^{2}, \pi^{2} n^{2}\right), \quad \gamma \in\left(\left(\pi^{2} n^{2}-\lambda^{\varepsilon}\right)^{1 / 2},\left(\pi^{2}(n+1)^{2}-\lambda^{\varepsilon}\right)^{1 / 2}\right) .
$$

Подчеркнем, что весовой показатель $\gamma$ подобран так, чтобы все волны (4), (17) попали в пространство $W_{-\gamma}^{1,0}\left(\Pi_{+}^{\varepsilon}\right)$. Следующее утверждение является конкретизацией результатов теории эллиптических задач в областях с цилиндрическими выходами на бесконечность (см. ключевые работы [18], [20], [23], [24] и, например, монографию [21], гл. 2, 5). Несмотря на то что эта теория обычно имеет дело с краевыми задачами в классической постановке, переход к интегральным тождествам вида (30) не встречает сколько-нибудь заметных препятствий (см., например, обзор [25]).

Теорема 4. Имеют место следующие утверждения.

1. Операторы $A_{ \pm \gamma}^{\varepsilon}\left(\lambda^{\varepsilon}\right)$ фредгольмовы, но терлют это свойство в концевых точках интервала (32) для показателя $\gamma$.

2. Если $f^{\varepsilon} \in W_{-\gamma}^{1,0}\left(\Pi_{+}^{\varepsilon}\right)^{*} \subset W_{\gamma}^{1,0}\left(\Pi_{+}^{\varepsilon}\right)^{*} u u^{\varepsilon} \in W_{-\gamma}^{1,0}\left(\Pi_{+}^{\varepsilon}\right)-$ решение задачи (30) с весовым показателем $\beta=-\gamma$, то справедливы представление

$$
u^{\varepsilon}=\widetilde{u}^{\varepsilon}+\sum_{ \pm} \sum_{k=1}^{n} c_{k}^{\varepsilon \pm} w_{k}^{ \pm}, \quad \widetilde{u}^{\varepsilon} \in W_{\gamma}^{1}\left(\Pi_{+}^{\varepsilon}\right), \quad c_{k}^{\varepsilon \pm} \in \mathbb{C},
$$

и оценка

$$
\left\|\widetilde{u}^{\varepsilon} ; W_{\gamma}^{1}\left(\Pi_{+}^{\varepsilon}\right)\right\|+\sum_{ \pm} \sum_{k=1}^{n}\left|c_{k}^{\varepsilon \pm}\right| \leqslant c_{\varepsilon}(\gamma)\left(\left\|f^{\varepsilon} ; W_{-\gamma}^{1,0}\left(\Pi_{+}^{\varepsilon}\right)^{*}\right\|+\left\|u^{\varepsilon} ; W_{-\gamma}^{1}\left(\Pi_{+}^{\varepsilon}\right)\right\|\right),
$$

в которой множитель $c_{\varepsilon}(\gamma)$ не зависит от $u^{\varepsilon} u f^{\varepsilon}$. 
3. Справедлива формула

$$
\text { Ind } A_{-\gamma}^{\varepsilon}\left(\lambda^{\varepsilon}\right)=\operatorname{Ind} A_{\gamma}^{\varepsilon}\left(\lambda^{\varepsilon}\right)+2 n,
$$

где Ind $A_{\gamma}^{\varepsilon}\left(\lambda^{\varepsilon}\right)=\operatorname{dim} \operatorname{ker} A_{\gamma}^{\varepsilon}\left(\lambda^{\varepsilon}\right)-\operatorname{dim} \operatorname{coker} A_{\gamma}^{\varepsilon}\left(\lambda^{\varepsilon}\right)-$ индекс фредгольмова оператора $A_{\gamma}^{\varepsilon}\left(\lambda^{\varepsilon}\right)$. При этом ядро $\operatorname{ker} A_{\gamma}^{\varepsilon}\left(\lambda^{\varepsilon}\right)$ оператора $(31)$, а значит, и коядро coker $A_{-\gamma}^{\varepsilon}\left(\lambda^{\varepsilon}\right)$ сопряженного оператора тривиальны, и поэтому

$$
\operatorname{dim} \operatorname{ker} A_{-\gamma}^{\varepsilon}\left(\lambda^{\varepsilon}\right)=\operatorname{dim} \operatorname{coker} A_{\gamma}^{\varepsilon}\left(\lambda^{\varepsilon}\right)=n
$$

ДокАзАтЕльство. Первые два утверждения, а также формула (35) для индекса - следствия общих результатов упомянутой выше теории (см., например, теорему 5.1.4 в [21]). Обратим внимание на несколько обстоятельств, присущих рассматриваемой задаче. Прежде всего, множитель $c_{\varepsilon}(\gamma)$ неограниченно возрастает при $\gamma \rightarrow\left(\pi^{2}-\lambda^{\varepsilon}\right)^{1 / 2}$ и поэтому, в частности, зависит от $\varepsilon$ для спектрального параметра $(5)$, близкого к порогу (эта зависимость уточняется в теореме 6). Если же

$$
\lambda \in\left(\pi^{2}(n-1)^{2}+\delta, \pi^{2} n^{2}-\delta\right)
$$

при некотором фиксированном $\delta>0$, то константу $c_{\varepsilon}(\gamma)$ можно взять общей для всех $\varepsilon \in\left(0, \varepsilon_{\delta}\right]$; здесь $\varepsilon_{\delta}>0$. Для проверки сказанного нужно сделать замену координат

$$
x \mapsto x^{\varepsilon}=\left(x_{1}^{\varepsilon}, x_{2}^{\varepsilon}\right)=\left(x_{1},\left(1+\varepsilon H\left(x_{1}\right)\right)^{-1}\left(x_{2}+\varepsilon H\left(x_{1}\right)\right)\right),
$$

которая придает малое возмущение оператору Гельмгольца в дифференциальном уравнении (6), и заметить, что оператор $A_{\gamma}^{\varepsilon}(\lambda)$ после замены (38) мало отличается от оператора $A_{\gamma}^{0}(\lambda)$ предельной задачи в полуполосе $\Pi_{+}^{0}$, для которой свойство независимости очевидно. Кроме того, у задачи в множестве $\Pi_{+}^{0}$ нет затухающих решений, т. е. $\operatorname{dim} \operatorname{ker} A_{\gamma}^{0}(\lambda)=0$. Благодаря почти тождественной замене координат (38) тривиальность ядра наследуется оператором $A_{\gamma}^{\varepsilon}(\lambda)$ при любом $\lambda^{\varepsilon} \in\left[0, \pi^{2} n^{2}\right]$.

ЗАмЕЧАНИЕ. Число $2 n$ в правой части соотношения (35) есть не что иное, как количество волн, отделенных в асимптотике (33). Формула (36) вытекает из проверенной формулы $\operatorname{dim} \operatorname{ker} A_{\gamma}^{\varepsilon}\left(\lambda^{\varepsilon}\right)=0$ и очевидного соотношения Ind $A_{-\gamma}^{\varepsilon}\left(\lambda^{\varepsilon}\right)=$ - Ind $A_{\gamma}^{\varepsilon}\left(\lambda^{\varepsilon}\right)$ для взаимно сопряженных операторов $A_{\gamma}^{\varepsilon}\left(\lambda^{\varepsilon}\right)$ и $A_{-\gamma}^{\varepsilon}\left(\lambda^{\varepsilon}\right)$. В подпространстве $\operatorname{ker} A_{-\gamma}^{\varepsilon}\left(\lambda^{\varepsilon}\right)$ существует базис $\left\{U_{1}^{\varepsilon}, \ldots, U_{n}^{\varepsilon}\right\}$ решений однородной задачи (30) с асимптотиками (18). В самом деле, если такого базиса нет, то найдется функция $U^{\varepsilon} \in \operatorname{ker} A_{-\gamma}^{\varepsilon}\left(\lambda^{\varepsilon}\right)$, для которой

$$
\begin{gathered}
U^{\varepsilon}=\widetilde{U}^{\varepsilon}+\sum_{k=1}^{n} K_{k}^{\varepsilon} w_{k}^{+}, \quad \tilde{U}^{\varepsilon} \in W_{\gamma}^{1}\left(\Pi_{+}^{\varepsilon}\right), \\
K_{k}^{\varepsilon \pm} \in \mathbb{C}, \quad|K|=\left(\left|K_{1}\right|^{2}+\cdots+\left|K_{n}\right|^{2}\right)^{1 / 2} \neq 0,
\end{gathered}
$$

а значит, подстановка ее в формулу Грина (15) приводит к противоречию $0=i|K|^{2}$.

СлЕДСТВИЕ. Число $\lambda^{\varepsilon}$ из соотношений (32) является собственным в том и только в том случае, когда $S_{n n}^{\varepsilon}=-1$. 
ДокАЗАТЕЛЬство. Поскольку согласно утверждению 3 теоремы 4 однородная $\left(f^{\varepsilon}=0\right)$ задача (30) не имеет решений $u^{\varepsilon} \in W_{\gamma}^{1,0}\left(\Pi_{+}^{\varepsilon}\right)$, осталось убедиться в следующем: если решение $u^{\varepsilon} \in H^{1,0}\left(\Pi_{+}^{\varepsilon}\right)$ допускает представление

$$
u^{\varepsilon}(x)=K_{n}^{\varepsilon} \sin \left(\pi n x_{2}\right) e^{-x_{1} \sqrt{\pi^{2} n^{2}-\lambda^{\varepsilon}}}+\widetilde{u}^{\varepsilon}(x), \quad \widetilde{u}^{\varepsilon} \in W_{\gamma}^{1}\left(\Pi_{+}^{\varepsilon}\right), \quad K_{n}^{\varepsilon \pm} \neq 0,
$$

то $S_{n n}^{\varepsilon}=-1$. В силу приведенного выше замечания включение $u^{\varepsilon} \in \operatorname{ker} A_{-\gamma}^{\varepsilon}$ означает, что $u^{\varepsilon}=C_{1}^{\varepsilon} U_{1}^{\varepsilon}+\cdots+C_{n}^{\varepsilon} U_{n}^{\varepsilon}$. В вытекающем из формулы (18) представлении

$u^{\varepsilon}=\sum_{k=1}^{n-1}\left(C_{k}^{\varepsilon} w_{k}^{-}+\sum_{j=1}^{n-1}\left(C_{k}^{\varepsilon} S_{j k}^{\varepsilon}+C_{n}^{\varepsilon} S_{j n}^{\varepsilon}\right) w_{j}^{+}\right)+C_{n}^{\varepsilon} w_{n}^{-}+\sum_{p=1}^{n} C_{p}^{\varepsilon} S_{p n}^{\varepsilon} w_{n}^{+}+\widehat{u}^{\varepsilon}, \quad \widehat{u}^{\varepsilon} \in W_{\gamma}^{1}\left(\Pi_{+}^{\varepsilon}\right)$,

должны обратиться в нуль коэффициенты при осциллирующих волнах $w_{k}^{ \pm}, k=$ $1, \ldots, n-1$, т. е. $C_{k}^{\varepsilon}=0$ и $S_{n k}^{\varepsilon}=S_{k n}^{\varepsilon}=0, k=1, \ldots, n-1$. Кроме того, равен нулю суммарный коэффициент при экспоненциальной волне (27), а значит, в силу уже установленных равенств имеем $1+S_{n n}^{\varepsilon}=0$, что и требовалось.

Достаточное условие [17] существования захваченной волны, сформулированное в теореме 2, превратилось в критерий исключительно благодаря отсутствию быстро затухающих решений однородной задачи (30) (утверждение 3 теоремы 4). В общей ситуации условие не является необходимым, и игнорирование этого факта приводит к ошибкам (некоторые из них обсуждаются в статье [19]).

Обозначим через $W_{\gamma}^{1,0}\left(\Pi_{+}^{\varepsilon}\right)^{\text {out }}$ пространство функций, представимых в виде

$$
u^{\varepsilon}=\widetilde{u}^{\varepsilon}+c_{1}^{\varepsilon} w_{1}^{+}+\ldots+c_{n}^{\varepsilon} w_{n}^{+},
$$

и снабдим его нормой

$$
\left\|u^{\varepsilon} ; W_{\gamma}^{1,0}\left(\Pi_{+}^{\varepsilon}\right)^{\text {out }}\right\|=\left\|\widetilde{u}^{\varepsilon} ; W_{\gamma}^{1}\left(\Pi_{+}^{\varepsilon}\right)\right\|+\left|c_{1}^{\varepsilon+}\right|+\cdots+\left|c_{n}^{\varepsilon+}\right| .
$$

Ясно, что $W_{\gamma}^{1,0}\left(\Pi_{+}^{\varepsilon}\right)^{\text {out }} \subset W_{-\gamma}^{1,0}\left(\Pi_{+}^{\varepsilon}\right)$. Согласно утверждению 2 теоремы 4 сужение $A_{\gamma}^{\varepsilon, \text { out }}$ оператора $A_{-\gamma}^{\varepsilon}$ на подпространство $W_{\gamma}^{1,0}\left(\Pi_{+}^{\varepsilon}\right)^{\text {out }}$ является расширением оператора $A_{\gamma}^{\varepsilon}$ и осуществляет непрерывное отображение

$$
A_{\gamma}^{\varepsilon, \text { out }}: \quad W_{\gamma}^{1,0}\left(\Pi_{+}^{\varepsilon}\right)^{\text {out }} \rightarrow W_{-\gamma}^{1,0}\left(\Pi_{+}^{\varepsilon}\right)^{*}
$$

Поскольку размерность факторпространства $W_{\gamma}^{1,0}\left(\Pi_{+}^{\varepsilon}\right)^{\text {out }} / W_{\gamma}^{1,0}\left(\Pi_{+}^{\varepsilon}\right)$ равна $n$, оператор (41) приобретает нулевой индекс. В силу наблюдений, сделанных в замечании и в комментариях к теореме 4, отображение (41) оказывается биекцией. Разложение

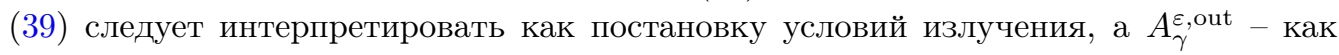
оператор задачи (2) с условиями излучения.

Присутствие в норме (40) модулей коэффициентов $c_{k}^{\varepsilon}$ позволяет заключить, что элементы расширенной матрицы рассеяния гладким образом зависят от параметров $\lambda \in\left(\pi^{2}(n-1)^{2}, \pi^{2} n^{2}\right)$ и $\varepsilon>0$, но предельные переходы $\lambda^{\varepsilon} \rightarrow \pi^{2} n^{2}-0$ и $\varepsilon \rightarrow+0$ требуют отдельной обработки, что и сделано в разделе 5 . 


\section{4. ФОРМАЛЬНЫЙ АСИМПТОТИЧЕСКИЙ АНАЛИЗ РАСШИРЕННОЙ МАТРИЦЫ РАССЕЯНИЯ}

При поиске собственного числа в виде (5) примем асимптотические анзацы

$$
\begin{gathered}
S_{n n}^{\varepsilon}=S_{n n}^{0}+\varepsilon S_{n n}^{\prime}+\widetilde{S}_{n n}^{\varepsilon}, \quad S_{\bullet n}^{\varepsilon}=\varepsilon^{1 / 2} S_{\bullet n}^{0}+\varepsilon^{3 / 2} S_{\bullet n}^{\prime}+\widetilde{S}_{\bullet n}^{\varepsilon}, \\
S_{\bullet \bullet}^{\varepsilon}=S_{\bullet \bullet}^{0}+\varepsilon S_{\bullet \bullet}^{\prime}+\widetilde{S}_{\bullet \bullet}^{\varepsilon}
\end{gathered}
$$

для составляющих матрицы (19) и анзацы

$$
U_{n}^{\varepsilon}(x)=\varepsilon^{-1 / 2} U_{n}^{0}(x)+\varepsilon^{1 / 2} U_{n}^{\prime}(x)+\widetilde{U}_{n}^{\varepsilon}(x), \quad U_{\bullet}^{\varepsilon}(x)=U_{\bullet}^{0}(x)+\varepsilon U_{\bullet}^{\prime}(x)+\widetilde{U}_{\bullet}^{\prime}(x)
$$

для специальных решений (18) задачи (6), (7). В данном разделе вычислим отдельные члены асимптотики, а в следующем оценим остатки.

Сначала займемся функцией $U_{n}^{\varepsilon}$, разложение (18) которой при учете соотношений (5) и (4), (17), а также (42) перепишем следующим образом:

$$
\begin{aligned}
& U_{n}^{\varepsilon}(x)=\left(4 \mu \varepsilon^{2}\right)^{-1 / 4} \sin \left(\pi n x_{2}\right)\left(e^{x_{1} \varepsilon(\sqrt{\mu}+\cdots)}+i e^{-x_{1} \varepsilon(\sqrt{\mu}+\cdots)}\right)+ \\
& +\left(4 \mu \varepsilon^{2}\right)^{-1 / 4}\left(S_{n n}^{0}+\varepsilon S_{n n}^{\prime}+\cdots\right) \sin \left(\pi n x_{2}\right)\left(e^{x_{1} \varepsilon(\sqrt{\mu}+\cdots)}-i e^{-x_{1} \varepsilon(\sqrt{\mu}+\cdots)}\right)+ \\
& +\varepsilon^{1 / 2} \sum_{k=1}^{n-1}\left(\pi^{2}\left(n^{2}-k^{2}\right)-\varepsilon^{2} \mu\right)^{-1 / 4}\left(S_{k n}^{0}+\cdots\right) \sin \left(\pi k x_{2}\right) e^{+i x_{1} \sqrt{\pi^{2}\left(n^{2}-k^{2}\right)-\varepsilon^{2} \mu+\cdots}}+\cdots= \\
& =\varepsilon^{-1 / 2}(4 \mu)^{-1 / 4} \sin \left(\pi n x_{2}\right)\left(1+i+S_{n n}^{0}(1-i)\right)+\varepsilon\left(S_{n n}^{\prime}+x_{1} \sqrt{\mu}\left(1-i+S_{n n}^{0}(1+i)\right)\right)+ \\
& +\varepsilon^{1 / 2} \sum_{k=1}^{n-1}\left(\pi^{2}\left(n^{2}-k^{2}\right)\right)^{-1 / 4} S_{k n}^{0} \sin \left(\pi k x_{2}\right) e^{+i x_{1} \pi \sqrt{n^{2}-k^{2}}}+\cdots
\end{aligned}
$$

Теперь многоточием обозначаются младшие члены, не существенные для формального асимптотического анализа, а при выводе соотношения (44) применена формула Тейлора относительно переменной $\varepsilon$.

При $\varepsilon=0$ множество $\Pi_{+}^{\varepsilon}$ трансформируется в полуполосу $\Pi_{+}^{0}=\mathbb{R}_{+} \times(0,1)$, и предельной для (6), (7) служит смешанная краевая задача

$$
\begin{aligned}
-\Delta u^{0}(x)= & \pi^{2} n^{2} u^{0}(x), \quad x \in \Pi_{+}^{0}, \quad \partial_{1} u^{0}\left(0, x_{2}\right)=0, \quad x_{2} \in(0,1), \\
& u^{0}\left(x_{1}, 0\right)=g\left(x_{1}\right), \quad u^{0}\left(x_{1}, 1\right)=0, \quad x_{1}>0 .
\end{aligned}
$$

Однородная $(g=0)$ задача (45) имеет $n$ решений

$$
\begin{gathered}
u_{n}^{0}(x)=\sin \left(\pi n x_{2}\right), \\
u_{k}^{0}(x)=\sin \left(\pi k x_{2}\right)\left(e^{i x_{1} \pi \sqrt{n^{2}-k^{2}}}+e^{-i x_{1} \pi \sqrt{n^{2}-k^{2}}}\right), \quad k=1, \ldots, n-1 .
\end{gathered}
$$

Главный член $U_{n}^{0}$ первого анзаца (43) должен удовлетворять задаче (45) при $g=0$ и согласно используемой процедуре сращивания (см., например, книгу [26]) вести себя на бесконечности так же, как и множитель при $\varepsilon^{-1 / 2}$ в правой части (44). Последнее обстоятельство позволит в разделе 5 построить глобальное асимптотическое приближение к функции $U_{n}^{\varepsilon}$ и обосновать асимптотики, найденные здесь формально. Итак, имеем

$$
U_{n}^{0}(x)=(4 \mu)^{-1 / 4}\left(1+i+S_{n n}^{0}(1-i)\right) u_{n}^{0}(x) .
$$


Функция (47) удовлетворяет исходной задаче (6), (7) всюду, кроме искривленного участка $\left\{x: x_{1} \in(0, l), x_{2}=-\varepsilon H\left(x_{1}\right)\right\}$ одной из сторон полуполосы $\Pi_{+}^{\varepsilon},-$ в краевом условии Дирихле на этом участке возникает невязка

$$
-U_{n}^{0}\left(x_{1},-\varepsilon H\left(x_{1}\right)\right)=\varepsilon H\left(x_{1}\right) \partial_{2} U_{n}^{0}\left(x_{1}, 0\right)+O\left(\varepsilon^{2}\right) .
$$

Решение $U_{n}^{\prime}$ задачи (45) с правой частью

$$
g\left(x_{1}\right)=(4 \mu)^{-1 / 4} \pi n H\left(x_{1}\right)\left(1+i+S_{n n}^{0}(1-i)\right)
$$

компенсирует главную часть невязки (48) и допускает следующее разложение при $k=n$ :

$$
\begin{aligned}
U_{n}^{\prime}(x)= & \left(b_{n}^{1} x_{1}+b_{n}^{0}\right) \sin \left(\pi n x_{2}\right)+\sum_{j=1}^{n-1} b_{j} \sin \left(\pi j x_{1}\right) e^{i x_{1} \pi \sqrt{n^{2}-j^{2}}}+ \\
& +O\left(e^{-x_{1} \pi \sqrt{2 n+1}}\right), \quad x_{1} \rightarrow+\infty
\end{aligned}
$$

Разложение (50) может быть получено при помощи метода Фурье (см., например, учебник [27]). Этот же метод позволяет заключить, что коэффициент $b_{n}^{0}$ произволен, а остальные находятся по формулам

$$
\begin{aligned}
b_{n}^{1} & =-(4 \mu)^{-1 / 4}\left(1+i+S_{n n}^{0}(1-i)\right) \eta_{n}, \\
b_{j} & =-(4 \mu)^{-1 / 4}\left(1+i+S_{n n}^{0}(1-i)\right) \eta_{j}, \quad j=1, \ldots, n-1,
\end{aligned}
$$

где величины $\eta_{j}$ вычислены по формуле (11) с заменой $H^{0} \mapsto H$. Для вывода соотношений (51) достаточно подставить в формулу Грина на прямоугольнике $\{x \in$ $\left.\Pi_{+}^{0}: x_{1}<R\right\}$ решение $U_{n}^{\prime}$ и поочередно функции (46), а затем, вычислив интегралы по сторонам, устремить длину $R$ к бесконечности.

Согласно процедуре сращивания поведение на бесконечности функции $U_{n}^{\prime}$, заданное соотношением (50), предопределено множителем при $\varepsilon^{1 / 2}$ в правой части (44), т. е. должны выполняться равенства

$$
\begin{gathered}
-\eta_{n}\left(1+i+S_{n n}^{0}(1-i)\right)=\sqrt{\mu}\left(1-i+S_{n n}^{0}(1+i)\right), \\
S_{j n}^{0}=i(4 \mu)^{-1 / 4}\left(\pi^{2}\left(n^{2}-j^{2}\right)\right)^{1 / 4}\left(1+i+S_{n n}^{0}(1-i)\right) \eta_{j}, \quad j=1, \ldots, n-1 .
\end{gathered}
$$

Отсюда выводим, что

$$
\begin{gathered}
S_{n n}^{0}=-\frac{2 \eta_{n} \sqrt{\mu}+i\left(\eta_{n}^{2}-\mu\right)}{\mu+\eta_{n}^{2}}, \\
S_{j n}^{0}=-\eta_{j}(4 \mu)^{1 / 4}\left(\pi^{2}\left(n^{2}-j^{2}\right)\right)^{1 / 4}(1+i) \frac{\eta_{n}-i \sqrt{\mu}}{\mu+\eta_{n}^{2}}, \quad j=1, \ldots, n-1 .
\end{gathered}
$$

Отметим два обстоятельства. Во-первых, $S_{n n}^{0}=-1$ при $\sqrt{\mu}=\eta_{n}>0$, т. е. стало понятно предположение (12). Во-вторых, остался неизвестным поправочный член $S_{n n}^{\prime}$ : точно так же на первом шаге процедуры, т. е. при определении функции (47), не удалось найти основной член $S_{n n}^{0}$ асимптотики элемента $S_{n n}^{\varepsilon}$ расширенной матрицы 
рассеяния. На следующем шаге можно было бы вычислить и величину $S_{n n}^{\prime}$, но она далее не требуется.

Построение асимптотики блока $S_{\bullet \bullet}^{\varepsilon}$ матрицы (19) значительно проще. Так, сходное с (44) разложение выглядит следующим образом:

$$
\begin{aligned}
U_{k}^{\varepsilon}(x)= & \left(\pi^{2}\left(n^{2}-k^{2}\right)\right)^{-1 / 4} \sin \left(\pi k x_{2}\right) e^{-i x_{1} \pi \sqrt{n^{2}-k^{2}}}+ \\
& +\sum_{j=1}^{n-1}\left(\pi^{2}\left(n^{2}-j^{2}\right)\right)^{-1 / 4}\left(S_{j k}^{0}+\varepsilon S_{j k}^{\prime}\right) \sin \left(\pi j x_{2}\right) e^{i x_{1} \pi \sqrt{n^{2}-j^{2}}}+ \\
& +(4 \mu)^{-1 / 4}\left(S_{n k}^{0}+\varepsilon S_{n k}^{\prime}\right) \sin \left(\pi n x_{2}\right)+\cdots .
\end{aligned}
$$

Сравнивая формулы (53) и (43), строим функцию $U_{k}^{0}$ из решений (46) однородной задачи (45):

$$
U_{k}^{0}(x)=\left(\pi^{2}\left(n^{2}-k^{2}\right)\right)^{-1 / 4} u_{k}^{0}(x)+(4 \mu)^{-1 / 4} S_{n k}^{0} u_{n}^{0}(x)
$$

При этом коэффициент $S_{k n}^{0}$ совпадает с коэффициентом $S_{n k}^{0}$ из (52) (см. соотношение (24)) и по необходимости приходится положить

$$
S_{j k}^{0}=\delta_{k, j}, \quad k, j=1, \ldots, n-1 .
$$

На этом формальный асимптотический анализ заканчивается, так как явный вид поправочных слагаемых $S_{n n}^{\prime}, S_{\bullet n}^{\prime}$ и $S_{\bullet \bullet}^{\prime}$ в формулах (42) не понадобится. Впрочем, асимптотическую процедуру можно было бы продолжить и построить полные асимптотические ряды для элементов расширенной матрицы рассеяния. Далее будут получены оценки

$$
\left|\widehat{S}_{n n}^{\varepsilon}\right|+\left|\widehat{S}_{\bullet \bullet}^{\varepsilon}\right| \leqslant c \varepsilon, \quad\left|\widehat{S}_{\bullet n}^{\varepsilon}\right| \leqslant c \varepsilon^{3 / 2}
$$

в которых упомянутые слагаемые присоединены к остаткам, т. е. $\widehat{S}_{n n}^{\varepsilon}=\widetilde{S}_{n n}^{\varepsilon}+\varepsilon S_{n n}^{\prime}$ и т. п.

\section{5. ОБОСНОВАНИЕ АСИМПТОТИКИ РАСШИРЕННОЙ МАТРИЦЫ РАССЕЯНИЯ}

Сначала получим равномерную относительно $\varepsilon$ оценку решения задачи (2) с условиями излучения (39) (см. конец раздела (3)) и спектральным параметром

$$
\lambda^{\varepsilon} \in\left[\pi^{2} n^{2}-C_{n} \varepsilon^{2}, \pi^{2} n^{2}-c_{n} \varepsilon^{2}\right], \quad C_{n}>c_{n}>0 .
$$

При $\varepsilon \rightarrow+0$ эта задача трансформируется в предельную задачу (45), у которой помимо осциллирующих волн

$$
\mathbf{w}_{k}^{0 \pm}(x)=\left(\pi^{2}\left(n^{2}-k^{2}\right)\right)^{-1 / 4} \sin \left(\pi k x_{2}\right) e^{ \pm i x_{1} \pi \sqrt{n^{2}-k^{2}}}, \quad k=1, \ldots, n-1,
$$

имеются стоячая и резонансные волны

$$
\mathbf{w}_{n}^{0}(x)=\sin \left(\pi n x_{2}\right), \quad \mathbf{w}_{n}^{1}(x)=x_{1} \sin \left(\pi n x_{2}\right) .
$$


Принцип Зоммерфельда не позволяет классифицировать последние, так как волновое число у волны $\mathbf{w}_{n}^{0}$ равно нулю. В статье [28] (см. также [21], гл. 5) введены линейные волны

$$
\mathbf{w}_{n}^{0 \pm}(x)=\left(x_{1} \mp i\right) \sin \left(\pi n x_{2}\right),
$$

которые вместе с волнами (57) удовлетворяют соотношениям (21) (проверяется непосредственным вычислением) и тем самым порождают унитарную $(n \times n)$-матрицу рассеяния на пороге. $\mathrm{K}$ сожалению, волны $\mathbf{w}_{n}^{0 \pm}$ не получаются предельным переходом из волн $w_{n}^{ \pm}$, что препятствует переносу "хороших" свойств оператора задачи (6), (7) с порога $\pi^{2} n^{2}$ на значения (56) спектрального параметра. Изменим определение (17) следующим образом:

$$
\begin{aligned}
& \mathbf{w}_{n}^{\varepsilon \pm}(x)= \frac{1}{2} \sin \left(\pi n x_{2}\right)\left(\frac{1}{\sqrt{\pi^{2} n^{2}-\lambda^{\varepsilon}}}\left(e^{x_{1} \sqrt{\pi^{2} n^{2}-\lambda^{\varepsilon}}}-e^{-x_{1} \sqrt{\pi^{2} n^{2}-\lambda^{\varepsilon}}}\right) \mp\right. \\
&\left.\mp i\left(e^{x_{1} \sqrt{\pi^{2} n^{2}-\lambda^{\varepsilon}}}+e^{-x_{1} \sqrt{\pi^{2} n^{2}-\lambda^{\varepsilon}}}\right)\right) .
\end{aligned}
$$

Можно убедиться в том, что новые экспоненциальные волны (58), а также прежние осциллирующие волны $\mathbf{w}_{k}^{\varepsilon \pm}=w_{k}^{ \pm}$из (4) удовлетворяют соотношениям (21). Кроме того, теперь волны $\mathbf{w}_{j}^{\varepsilon \pm}, j=1, \ldots, n$, плавно переходят в волны $\mathbf{w}_{j}^{0 \pm}$ на конечном участке волновода: нетрудно убедиться в справедливости неравенств

$$
\left\|\mathbf{w}_{j}^{\varepsilon \pm}-\mathbf{w}_{j}^{0 \pm} ; H^{1}\left(\Pi_{+}^{0}(R)\right)\right\| \leqslant c_{R} \varepsilon, \quad j=1, \ldots, n, \quad R>0 .
$$

В следующей теореме число $\lambda^{\varepsilon}$ по-прежнему взято из $(56)$, однако $\varepsilon \in\left[0, \varepsilon_{n}\right)$ при некотором $\varepsilon_{n}>0$, т. е. в рассмотрение включен и предельный случай $\varepsilon=0$, $\lambda^{0}=\pi^{2} n^{2}$.

ТеОРема 5. Имеют место следующие утверждения.

1. При $\varepsilon \in\left[0, \varepsilon_{n}\right)$ и любой правой части $f^{\varepsilon} \in W_{-\gamma}^{1,0}\left(\Pi_{+}^{\varepsilon}\right)^{*}$ задача (6), (7) имеет единственное решение $\mathbf{u}^{\varepsilon} \in W_{-\gamma}^{1,0}\left(\Pi_{+}^{\varepsilon}\right)$, допускающее разложение

$$
\mathbf{u}^{\varepsilon}=\widetilde{\mathbf{u}}^{\varepsilon}+\mathbf{c}_{1}^{\varepsilon} \mathbf{w}_{1}^{+}+\cdots+\mathbf{c}_{n-1}^{\varepsilon} \mathbf{w}_{n-1}^{+}+\mathbf{c}_{n}^{\varepsilon} \mathbf{w}_{n}^{+},
$$

т.е. подчиненное условиям излучения; здесь $\widetilde{\mathbf{u}}^{\varepsilon} \in W_{\gamma}^{1}\left(\Pi_{+}^{\varepsilon}\right)$ u $\mathbf{c}_{j}^{\varepsilon} \in \mathbb{C}$. Множитель $c(\gamma)$ в оценке

$$
\left\|\widetilde{\mathbf{u}}^{\varepsilon} ; W_{\gamma}^{1}\left(\Pi_{+}^{\varepsilon}\right)\right\|+\left|\mathbf{c}_{1}^{\varepsilon}\right|+\cdots+\left|\mathbf{c}_{n-1}^{\varepsilon}\right|+\left|\mathbf{c}_{n}^{\varepsilon}\right| \leqslant c(\gamma)\left\|f^{\varepsilon} ; W_{-\gamma}^{1,0}\left(\Pi_{+}^{\varepsilon}\right)^{*}\right\|
$$

не зависит от малого параметра $\varepsilon \in\left[0, \varepsilon_{n}\right)$.

2. У задачи (6), (7) есть решения $\mathbf{U}_{1}^{\varepsilon}, \ldots, \mathbf{U}_{n}^{\varepsilon}$, удовлетворяющие требованиям

$$
\mathbf{U}_{p}^{\varepsilon}=\mathbf{w}_{p}^{\varepsilon-}+\sum_{q=1}^{n} \mathbf{S}_{q p}^{\varepsilon} \mathbf{w}_{q}^{\varepsilon+}+\widetilde{\mathbf{U}}_{p}^{\varepsilon}
$$

при этом $\widetilde{\mathbf{U}}_{p}^{\varepsilon} \in W_{\gamma}^{1}\left(\Pi_{+}^{\varepsilon}\right)$, a $(n \times n)$-матрица $\mathbf{S}^{\varepsilon}$, составленная из коэффициентов разложений в (62), является унитарной и подчинена соотношениям

$$
\left\|\mathbf{S}^{\varepsilon}-\mathbf{S}^{0} ; \mathbb{C}^{n \times n}\right\| \leqslant c \varepsilon, \quad \mathbf{S}^{0}=\operatorname{diag}\{1, \ldots, 1,-1\} .
$$


ДоказАтельство. Оба утверждения теоремы при $\varepsilon=0$ и в случае задачи в прямой полуполосе $\Pi_{+}^{0}$, установлены в статье [28] (см. также [21], § 5.3), однако все нужные для их проверки соображения уже приводились в разделах 2 и 3 . Отметим лишь, что диагональная матрица $\mathbf{S}^{0}$ из (63) возникла при анализе решений (46) однородной задачи (45): ее элементы, равные единице, в количестве $n-1$ штук суть коэффициенты при уходящих волнах $\mathbf{w}_{k}^{0+}$ в решениях $u_{k}^{0}, k=1, \ldots, n-1$, из формулы (46), а решение

$$
2 i u_{n}^{0}(x)=2 i \sin \left(\pi n x_{2}\right)=\left(\left(x_{1}+i\right)-\left(x_{1}-i\right)\right) \sin \left(\pi n x_{2}\right)
$$

породило последний элемент -1 .

Переход к $\varepsilon>0$ осуществляется при помощи следующих соображений: во-первых, замена координат (38) и отклонение (56) спектрального параметра от порогового значения $\pi^{2} n^{2}$ привносят в дифференциальный оператор задачи малые возмущения, во-вторых, соотношения (59) показывают, что замена $\mathbf{w}_{p}^{0 \pm} \mapsto \mathbf{w}_{p}^{\varepsilon \pm}$ отделенных членов асимптотики порождает малое изменение данных задачи для асимптотического остатка $\widetilde{\mathbf{u}}^{\varepsilon}$. Иными словами, утверждения для $\varepsilon \in\left(0, \varepsilon_{n}\right)$ вытекают из утверждений для $\varepsilon=0$.

Ближайшая цель состоит в том, чтобы привести решение (60) к виду (39). Положим

$$
u^{\varepsilon}=\mathbf{u}^{\varepsilon}+\mathbf{b}_{n}^{\varepsilon} \mathbf{U}_{n}^{\varepsilon}
$$

и подберем коэффициент $\mathbf{b}_{n}^{\varepsilon}$ так, чтобы при некотором $c_{n}^{\varepsilon}$ выполнялось соотношение

$$
c_{n}^{\varepsilon} w_{n}^{+}=\mathbf{c}_{n}^{\varepsilon} \mathbf{w}_{n}^{\varepsilon+}+\mathbf{b}_{n}^{\varepsilon}\left(\mathbf{w}_{n}^{\varepsilon-}+\mathbf{S}_{n n}^{\varepsilon} \mathbf{w}_{n}^{\varepsilon+}\right) .
$$

В результате благодаря равенствам $w_{k}^{+}=\mathbf{w}_{k}^{\varepsilon+}, k=1, \ldots, n-1$, и формулам (60), (62) сумма (65) приобретет вид (39) и превратится в искомое решение задачи (6), (7) с условиями излучения, выбранными в разделе 3.

Собирая множители при растущих и затухающих волнах в соответствии с определениями (17) и (58), выводим из соотношения (66) следующую систему уравнений для неизвестных $c_{n}^{\varepsilon}$ и $\mathbf{b}_{n}^{\varepsilon}$ :

$$
\begin{aligned}
& \left|4\left(\pi^{2} n^{2}-\lambda^{\varepsilon}\right)\right|^{-1 / 4} c_{n}^{\varepsilon}-\frac{1}{2}\left(\left(\pi^{2} n^{2}-\lambda^{\varepsilon}\right)^{-1 / 2}+i\right)+ \\
& \left.\quad+\mathbf{S}_{n n}^{\varepsilon}\left(\left(\pi^{2} n^{2}-\lambda^{\varepsilon}\right)^{-1 / 2}-i\right)\right) \mathbf{b}_{n}^{\varepsilon}=\frac{1}{2}\left(\left(\pi^{2} n^{2}-\lambda^{\varepsilon}\right)^{-1 / 2}-i\right) \mathbf{c}_{n}^{\varepsilon} \\
& -i\left|4\left(\pi^{2} n^{2}-\lambda^{\varepsilon}\right)\right|^{-1 / 4} c_{n}^{\varepsilon}+\frac{1}{2}\left(\left(\pi^{2} n^{2}-\lambda^{\varepsilon}\right)^{-1 / 2}-i\right)+ \\
& \left.\quad+\mathbf{S}_{n n}^{\varepsilon}\left(\left(\pi^{2} n^{2}-\lambda^{\varepsilon}\right)^{-1 / 2}+i\right)\right) \mathbf{b}_{n}^{\varepsilon}=-\frac{1}{2}\left(\left(\pi^{2} n^{2}-\lambda^{\varepsilon}\right)^{-1 / 2}+i\right) \mathbf{c}_{n}^{\varepsilon}
\end{aligned}
$$

Определитель

$$
\operatorname{det} \mathbf{M}^{\varepsilon}=\frac{1}{2}(1-i)\left|4\left(\pi^{2} n^{2}-\lambda^{\varepsilon}\right)\right|^{-1 / 4}\left(\left(\pi^{2} n^{2}-\lambda^{\varepsilon}\right)^{-1 / 2}\left(\mathbf{S}_{n n}^{\varepsilon}+1\right)-\left(\mathbf{S}_{n n}^{\varepsilon}-1\right)\right)
$$

матрицы $\mathbf{M}^{\varepsilon}$ системы (67) удовлетворяет неравенствам

$$
\begin{aligned}
0<\mathbf{c} & \leqslant \varepsilon^{1 / 2}\left|\operatorname{det} \mathbf{M}^{\varepsilon}\right|= \\
& =\frac{1}{2} \varepsilon^{1 / 2}\left|4\left(\pi^{2} n^{2}-\lambda^{\varepsilon}\right)\right|^{-1 / 4}\left|\left(\pi^{2} n^{2}-\lambda^{\varepsilon}\right)^{-1 / 2}\left(\mathbf{S}_{n n}^{\varepsilon}+1\right)-\left(\mathbf{S}_{n n}^{\varepsilon}-1\right)\right| \leqslant \mathbf{C} .
\end{aligned}
$$


При этом учтено соотношение (56) для $\lambda^{\varepsilon}$, а также формулы (63) для $\mathbf{S}^{\varepsilon}$, означающие, что $\mathbf{S}_{n n}^{\varepsilon}-1=-2+O(\varepsilon)$ и

$$
2 \operatorname{Re}\left(\mathbf{S}_{n n}^{\varepsilon}+1\right)=\left|\mathbf{S}_{n n}^{\varepsilon}+1\right|^{2}-\left|\mathbf{S}_{\bullet n}^{\varepsilon}\right|^{2}=O\left(\varepsilon^{2}\right) \quad \Rightarrow \quad \operatorname{Re}\left(\varepsilon^{-1}\left(\mathbf{S}_{n n}^{\varepsilon}+1\right)\right)=O(\varepsilon) .
$$

Система (67) разрешима, а оценка

$$
\varepsilon^{1 / 2}\left|c_{n}^{\varepsilon}\right|+\varepsilon\left|\mathbf{b}_{n}^{\varepsilon}\right| \leqslant c\left|\mathbf{c}_{n}^{\varepsilon}\right|
$$

ее решения выводится следующим образом. Сначала по правилу Крамера находим, чTо

$$
\left|c_{n}^{\varepsilon}\right| \leqslant c\left|\operatorname{det} \mathbf{M}^{\varepsilon}\right|^{-1}\left(1+\left(\pi^{2} n^{2}-\lambda^{\varepsilon}\right)^{-1 / 2}\right)\left|\mathbf{c}_{n}^{\varepsilon}\right| \leqslant c \varepsilon^{-1 / 2}\left|\mathbf{c}_{n}^{\varepsilon}\right|,
$$

а затем, сложив строки системы, выражаем $\mathbf{b}_{n}^{\varepsilon}$ через $c_{n}^{\varepsilon}, \mathbf{c}_{n}^{\varepsilon}$ и получаем, что

$$
\left|\mathbf{b}_{n}^{\varepsilon}\right| \leqslant c\left(\left|\mathbf{c}_{n}^{\varepsilon}\right|+\varepsilon^{-1 / 2}\left|c_{n}^{\varepsilon}\right|\right) \leqslant c \varepsilon^{-1}\left|\mathbf{c}_{n}^{\varepsilon}\right|
$$

Осталось заметить, что в силу $(61),(63)$ и (68) для коэффициентов $c_{1}^{\varepsilon}, \ldots, c_{n-1}^{\varepsilon}$ в разложении (39) решения (65) верны соотношения

$$
c_{k}^{\varepsilon}=\mathbf{c}_{k}^{\varepsilon}+\mathbf{S}_{k n}^{\varepsilon} \mathbf{b}_{n}^{\varepsilon}, \quad\left|c_{k}^{\varepsilon}\right| \leqslant c\left(\left|\mathbf{c}_{k}^{\varepsilon}\right|+\varepsilon\left|\mathbf{b}_{n}^{\varepsilon}\right|\right) \leqslant c\left(\left|\mathbf{c}_{k}^{\varepsilon}\right|+\left|\mathbf{c}_{n}^{\varepsilon}\right|\right) \leqslant c\left\|f^{\varepsilon} ; W_{-\gamma}^{0}\left(\Pi_{+}^{\varepsilon}\right)^{*}\right\| .
$$

Для $W_{\gamma}^{1}\left(\Pi_{+}^{\varepsilon}\right)$-нормы остатка $\widetilde{u}^{\varepsilon}=\widetilde{\mathbf{u}}^{\varepsilon}+\mathbf{b}_{n}^{\varepsilon} \widetilde{\mathbf{U}}_{n}^{\varepsilon}$ возникает мажоранта $O\left(\varepsilon^{-1}\right)$, но можно убедиться в том, что $\left\|\widetilde{\mathbf{U}}{ }_{n}^{\varepsilon} ; W_{\gamma}^{1}\left(\Pi_{+}^{\varepsilon}\right)\right\|=O(\varepsilon)$, так как $\widetilde{\mathbf{U}}_{n}^{0}=0$ согласно формуле (64), и вывести правильную оценку для $\widetilde{u}^{\varepsilon}$, которая приведена в очередной теореме, но далее востребована не будет.

Tеорема 6. Пусть $f^{\varepsilon} \in W_{-\gamma}^{1,0}\left(\Pi_{+}^{\varepsilon}\right)^{*}$, а число $\lambda^{\varepsilon}$ задано в (56). Тогда задача (30) с $\beta=-\gamma$ имеет единственное решение $u^{\varepsilon}$, допускающее представление (39), $u$ справедлива оценка

$$
\left\|\widetilde{u}^{\varepsilon} ; W_{\gamma}^{1}\left(\Pi_{+}^{\varepsilon}\right)\right\|+\left|c_{1}^{\varepsilon}\right|+\cdots+\left|c_{n-1}^{\varepsilon}\right|+\varepsilon^{1 / 2}\left|c_{n}^{\varepsilon}\right| \leqslant c\left\|f^{\varepsilon} ; W_{-\gamma}^{1,0}\left(\Pi_{+}^{\varepsilon}\right)^{*}\right\|,
$$

в которой множитель с не зависит от малого параметра $\varepsilon \in\left(0, \varepsilon_{n}\right), \varepsilon_{n}>0$.

Теперь можно приступить к обоснованию асимптотических разложений, представленных в разделе 4. Применим конструкцию, присущую методу составных разложений (см. [29], гл. 2, 5), и в качестве глобального асимптотического приближения к решению $U_{n}^{\varepsilon}$ задачи (6), (7) возьмем выражение

$$
\begin{aligned}
\mathcal{U}_{n}^{\varepsilon}(x)= & \chi_{\infty}\left(x_{1}\right)\left(w_{n}^{-}(x)+S_{n n}^{0} w_{n}^{+}(x)+\varepsilon^{1 / 2} \sum_{k=1}^{n-1} S_{k n}^{0} w_{k}^{+}(x)\right)+ \\
& +\varepsilon^{-1 / 2} \widetilde{U}_{n}^{0}(x)+\varepsilon^{1 / 2} \widetilde{U}_{n}^{\prime}(x)-\varepsilon^{3 / 2} \widehat{U}_{n}^{\varepsilon}(x) .
\end{aligned}
$$

Приведем пояснения. В первую очередь отметим, что $\chi_{\infty}-$ гладкая срезающая функция, равная единице при $x_{1}>2 l$ и нулю при $x_{1}<l, S_{n n}^{0}$ и $S_{k n}^{0}-$ величины $(52)$, а $w_{j}^{ \pm}$- волны, определенные формулами (4) и (17) по спектральному параметру (56). Эти волны обращаются в нуль на боковых сторонах полуполосы $\Pi_{+}^{0}$, т. е. первое слагаемое в правой части $(70)$, обращающееся в нуль при $x_{1}<l$, оставляет невязку 
только в уравнении Гельмгольца. Эта невязка, имеющая носитель в прямоугольнике $\mathbb{Q}_{l}=[l, 2 l] \times[0,1]$, содержит коммутатор оператора Лапласа и срезающей функции. При помощи аналогичной (44) формулы Тейлора она представима в виде

$$
\begin{array}{r}
{\left[\Delta, \chi_{\infty}\right]\left(w_{n}^{\varepsilon}(x)+S_{n n}^{0} w_{n}^{+}(x)+\varepsilon^{1 / 2} \sum_{k=1}^{n-1} S_{k n}^{0} w_{k}^{+}(x)\right)=} \\
=\left[\Delta, \chi_{\infty}\right]\left(\varepsilon^{-1 / 2} B_{n}^{0}(x)+\varepsilon^{1 / 2} B_{n}^{\prime}(x)\right)+O\left(\varepsilon^{3 / 2}\right) .
\end{array}
$$

Благодаря проведенному сращиванию функции

$$
\begin{aligned}
B_{n}^{0}(x)= & (4 \mu)^{-1 / 4}\left(1+i+S_{n n}^{0}(1-i)\right) \sin \left(\pi n x_{2}\right) \\
B_{n}^{\prime}(x)= & (4 \mu)^{-1 / 4} x_{1} \sqrt{\mu}\left(1-i+S_{n n}^{0}(1+i)\right) \sin \left(\pi n x_{2}\right)+ \\
& +\sum_{k=1}^{n-1}\left(\pi^{2}\left(n^{2}-k^{2}\right)\right)^{-1 / 4} S_{k n}^{0} \sin \left(\pi k x_{2}\right) e^{i x_{1} \sqrt{n^{2}-k^{2}}}
\end{aligned}
$$

одновременно являются незатухающими членами разложений на бесконечности решений $U_{n}^{0}$ и $U_{n}^{\prime}$ (см. формулы $(47)$ и $(50)$, причем в последней мы положили $b_{n}^{0}=0$, а остальные коэффициенты взяли из (51)). Во избежание дублирования эти функции пришлось удалить из упомянутых решений:

$$
\widetilde{U}_{n}^{0}=U_{n}^{0}-\chi_{\infty} B_{n}^{0}, \quad \widetilde{U}_{n}^{\prime}=U_{n}^{\prime}-\chi_{\infty} B_{n}^{\prime} .
$$

При этом функция $\widetilde{U}_{n}^{\prime}$ экспоненциально затухает при $x_{1} \rightarrow+\infty$, а $\widetilde{U}_{n}^{0}$ вообще имеет компактный носитель. Невязка суммы $\varepsilon^{-1 / 2} U_{n}^{0}+\varepsilon^{1 / 2} U_{n}^{\prime}$ в уравнении (6) равна

$$
\left(\lambda^{\varepsilon}-\pi^{2}\right)\left(\varepsilon^{-1 / 2} U_{n}^{0}(x)+\varepsilon^{1 / 2} U_{n}^{\prime}(x)\right)-\left[\Delta, \chi_{\infty}\right]\left(\varepsilon^{-1 / 2} B_{n}^{0}(x)+\varepsilon^{1 / 2} B_{n}^{\prime}(x)\right) .
$$

Второе слагаемое взаимно уничтожается с аналогичным членом в (71), а первое в силу (56) приобретает порядок $\varepsilon^{3 / 2}$.

Краевое условие Неймана на торце $\varpi_{0}^{\varepsilon}$ соблюдено. Осталось позаботится об условии Дирихле на искривленном участке границы $\partial \Pi_{+}^{\varepsilon}$. Согласно определению (31) правой части $g$ задачи для $U_{n}^{\prime}$ имеем

$$
\begin{aligned}
& \left.\varepsilon^{-1 / 2}\left(U_{n}^{0}(x)+\varepsilon U_{n}^{\prime}\left(x_{1}\right)\right)\right|_{x_{2}=-\varepsilon H\left(x_{1}\right)}= \\
& \quad=\varepsilon^{-1 / 2}\left(U_{n}^{0}(x)-x_{2} \partial_{2} U_{n}^{0}\left(x_{1}, 0\right)+\left.\varepsilon\left(U_{n}^{\prime}(x)-U_{n}^{\prime}\left(x_{1}, 0\right)\right)\right|_{x_{2}=-\varepsilon H\left(x_{1}\right)}=O\left(\varepsilon^{3 / 2}\right) .\right.
\end{aligned}
$$

Отметим, что благодаря введенному условию ${ }^{2)} H(0)=0$ решение $U_{n}^{\prime}$ оказывается дважды непрерывно дифференцируемым вплоть до угловых точек (см., например, монографию [2], гл. 2). Поэтому невязку (72) удается компенсировать функцией $\varepsilon^{3 / 2} \widehat{U}_{n}^{\varepsilon}$ с компактным носителем, у которой $\left\|\widehat{U}_{n}^{\varepsilon} ; H^{1}\left(\Pi_{+}^{\varepsilon}\right)\right\|=O(1)$. Подчеркнем, что эта функция порождает малую невязку в условии Неймана на торце.

Подытоживая приведенные рассуждения и выкладки, обнаруживаем, что разность $\mathcal{R}_{n}^{\varepsilon}=\mathcal{U}_{n}^{\varepsilon}-U_{n}^{\varepsilon} \in W_{\gamma}^{1,0}\left(\Pi_{+}^{\varepsilon}\right)^{\text {out }}$ оказывается решением задачи (30), в правую

2)При $H(0) \neq 0$ теорема 1 также справедлива (см. раздел 9), но конструкция (70) требует значительного усложнения. 
часть которой помещен функционал $\mathcal{F}_{n}^{\varepsilon}$ с $W_{-\gamma}^{1,0}\left(\Pi_{+}^{\varepsilon}\right)^{*}$-нормой $O\left(\varepsilon^{3 / 2}\right)$. Таким образом, в силу оценки (69) коэффициенты $\mathcal{C}_{j}^{\varepsilon}$ при волнах $w_{j}^{+}$в разложении $(39)$ функции $\mathcal{R}_{n}^{\varepsilon}$ удовлетворяют неравенствам

$$
\left|\mathcal{C}_{k}^{\varepsilon}\right| \leqslant c \varepsilon^{3 / 2}, \quad k=1, \ldots, n-1, \quad\left|\mathcal{C}_{n}^{\varepsilon}\right| \leqslant c \varepsilon .
$$

Заметим, что названные коэффициенты совпадают с величинами $\widehat{S}_{j n}^{\varepsilon}$, которые в разделе 4 и было обещано оценить. Поскольку остальные решения $U_{1}^{\varepsilon}, \ldots, U_{n-1}^{\varepsilon}$ обрабатываются гораздо проще, формулируем полученный результат.

Теорема 7. Для разностей $\widehat{S}_{n n}^{\varepsilon}=S_{n n}^{\varepsilon}-S_{n n}^{0}, \widehat{S}_{k k}^{\varepsilon}=S_{k k}^{\varepsilon}-S_{k k}^{0} u \widehat{S}_{j k}^{\varepsilon}=S_{j k}^{\varepsilon}-$ $\varepsilon^{1 / 2} S_{j k}^{0}$, где $j, k=1, \ldots, n-1 u j \neq k$, верны формулы (55).

\section{6. ИДЕНТИФИКАЦИЯ ЗАХВАЧЕННОЙ ВОЛНЫ}

Примем условия (10)-(13) и убедимся в том, что соотношения

$$
\operatorname{Im} S_{n n}^{\varepsilon}=0, \quad \operatorname{Re} S_{k n}^{\varepsilon}=0, \quad k=0, \ldots, n-1,
$$

гарантируют равенство $S_{n n}^{\varepsilon}=-1$, т. е. по теореме 2 собственное число и захваченная волна существуют.

В соответствии с комментарием к первой из формул (52) положим

$$
\mu=\eta_{n, 0}^{2}+\tau_{0},
$$

а параметр $\tau_{0}$ также подчиним включению (14). Таким образом, согласно (52), (54) и (55) находим

$$
\left|S_{n n}^{\varepsilon}+1\right| \leqslant c(\varepsilon+|\vec{\tau}|), \quad\left|S_{k j}^{\varepsilon}-\delta_{k, j}\right| \leqslant c(\varepsilon+|\vec{\tau}|)
$$

где $|\vec{\tau}|=\left|\tau_{0}\right|+\left|\tau_{1}\right|+\cdots+\left|\tau_{n-1}\right|$. Кроме того, соотношения (73) и (24) показывают, что $S_{n k}^{\varepsilon}=S_{k n}^{\varepsilon}=-\overline{S_{k n}^{\varepsilon}}$. Благодаря ортогональности столбцов унитарной матрицы $S^{\varepsilon}$ имеем

$$
\begin{aligned}
0 & =S_{1 k}^{\varepsilon} \overline{S_{1 n}^{\varepsilon}}+\cdots+S_{n-1 k}^{\varepsilon} \overline{S_{n-1 n}^{\varepsilon}}+S_{n k}^{\varepsilon} \overline{S_{n n}^{\varepsilon}}= \\
& =-2 S_{k n}^{\varepsilon}+O\left((\varepsilon+|\vec{\tau}|)\left(\left|S_{1 n}^{\varepsilon}\right|^{2}+\cdots+\left|S_{n-1 n}^{\varepsilon}\right|^{2}\right)^{1 / 2}\right), \quad k=1, \ldots, n-1 .
\end{aligned}
$$

Отсюда вытекает, что $S_{1 n}^{\varepsilon}=\cdots=S_{n-1 n}^{\varepsilon}=0$ при малых $\varepsilon$ и $|\vec{\tau}|$, а значит, $\left|S_{n n}^{\varepsilon}\right|=1$ и $S_{n n}^{\varepsilon}=-1$ в силу $(74)$.

Вспоминая асимптотические формулы (52) и представления (10), (74) при учете условий (13) перепишем соотношения (73) следующим образом:

$$
\begin{aligned}
\tau_{0} & =\eta_{n}^{2}-\eta_{n, 0}^{2}-\left(\mu+\eta_{n}^{2}\right) \operatorname{Im} \widehat{S}_{n n}^{\varepsilon}, \\
\tau_{j} & =-(4 \mu)^{-1 / 4} \frac{\eta_{n}^{2}+\mu}{\eta_{n}+\sqrt{\mu}}\left(\pi^{2}\left(n^{2}-j^{2}\right)\right)^{-1 / 4} \varepsilon^{-1 / 2} \operatorname{Re} \widehat{S}_{n j}^{\varepsilon}, \quad j=1, \ldots, n-1,
\end{aligned}
$$

или кратко

$$
\vec{\tau}=T[\vec{\tau}], \quad \vec{\tau}=\left(\tau_{0}, \tau_{1}, \ldots, \tau_{n-1}\right) \in \mathbb{R}^{n}
$$


Подчеркнем, что величины $\mu$ и $\eta_{n}$ в (75) зависят от вектора $\vec{\tau}$, причем $\left|\eta_{n}^{2}-\eta_{n, 0}^{2}\right| \leqslant$ $c|\vec{\tau}|$. Кроме того, множитель $c$ в оценках (55) можно зафиксировать общим для всех $\varepsilon \in\left(0, \varepsilon_{0}\right]$ и $\vec{\tau} \in B_{\rho_{0}}$; здесь $B_{\rho_{0}}$ - шар в $\mathbb{R}^{n}$ с радиусом $\rho_{0}$, а $\varepsilon_{0}$ и $\rho_{0}$ - некоторые положительные числа. В итоге благодаря оценкам (55) последних сомножителей в (75) непрерывный нелинейный оператор $T$ при некотором $K>0$ переводит шар $B_{\varepsilon K}$ в часть того же шара, т. е. согласно принципу Шаудера (теореме о неподвижной точке) уравнение (76) имеет решение $\vec{\tau} \in B_{\varepsilon K}$, т. е. теорема 1 доказана в части существования.

\section{7. О ЕДИНСТВЕННОСТИ СОБСТВЕННОГО ЧИСЛА}

Отсутствие собственных чисел на интервале (37) при любом фиксированном $\delta$ и малом $\varepsilon>0$ можно проверить, построив асимптотику расширенной матрицы рассеяния. В самом деле, предельная задача

$$
\begin{gathered}
-\Delta u(x)=\lambda u(x), \quad x \in \Pi_{+}^{0}, \\
\partial_{1} u\left(0, x_{2}\right)=0, \quad x_{2} \in(0,1), \quad u\left(x_{1}, 0\right)=u\left(x_{1}, 1\right)=0, \quad x_{1}>0,
\end{gathered}
$$

имеет следующие решения:

$$
\begin{aligned}
& u_{k}(x)=\sin \left(\pi k x_{2}\right)\left(e^{-i x_{1} \sqrt{\lambda-\pi^{2} k^{2}}}+e^{i x_{1} \sqrt{\lambda-\pi^{2} k^{2}}}\right), \quad k=1, \ldots, n-1, \\
& u_{n}(x)=\sin \left(\pi n x_{2}\right)\left(e^{x_{1} \sqrt{\pi^{2} n^{2}-\lambda}}+e^{-x_{1} \sqrt{\pi^{2} n^{2}-\lambda}}\right)
\end{aligned}
$$

поэтому

$$
S^{0}=\operatorname{diag}\{1, \ldots, 1,-i\} .
$$

Повторив со значительными упрощениями (в частности, потому, что постоянная $c_{\varepsilon}(\gamma)$ в неравенстве $(34)$ заведомо не зависит от $\varepsilon$ ) рассуждения из раздела 5 , получаем соотношение $\left\|S^{\varepsilon}-S^{0} ; \mathbb{C}^{n \times n}\right\| \leqslant C \varepsilon$. Таким образом, критерий $S_{n n}^{\varepsilon}=-1$ существования захваченной волны не выполнен при $\varepsilon \in\left(0, \varepsilon_{n}(\delta)\right)$ и некотором $\varepsilon_{n}(\delta)>0$.

Если для некоторой бесконечно малой последовательности $\left\{\varepsilon_{m}\right\}$ на полуинтервале $\left[\pi^{2}(n-1)^{2}, \pi^{2} n^{2}\right)$ нашлись два собственных числа задач (6), (7) с параметром $\varepsilon_{m}$, то в силу теоремы 1 , уже доказанной в части существования, возможны две ситуации: имеют место сходимости

$$
\lambda_{1}^{\varepsilon_{m}} \rightarrow \pi^{2}(n-1)^{2}+0
$$

или

$$
\lambda_{q}^{\varepsilon_{m}} \rightarrow \pi^{2} n^{2}-0, \quad q=1,2
$$

(может быть, вдоль подпоследовательности). Покажем, что эти соотношения ложны.

Обозначим через $u_{q}^{\varepsilon_{m}} \in H^{1,0}\left(\Pi_{+}^{\varepsilon_{m}}\right)$ собственные функции, отвечающие собственным числам (81), нормируем их условиями

$$
\left\|u_{q}^{\varepsilon_{m}} ; L_{2}\left(\mathbb{Q}_{l}\right)\right\|=1, \quad \mathbb{Q}_{l}=[l, 2 l] \times[0,1],
$$

и заметим, что

$$
\left(u_{1}^{\varepsilon_{m}}, u_{2}^{\varepsilon_{m}}\right)_{\Pi_{+}^{\varepsilon_{m}}}=0 .
$$


Функции $u_{q}^{\varepsilon_{m}}$ удовлетворяют задаче (30) с $f_{q}^{\varepsilon_{m}}=0$ и принадлежат пространству $W_{-\gamma}^{1,0}\left(\Pi_{+}^{\varepsilon_{m}}\right)$. Ввиду возможности произвести почти тождественную замену координат (38) множитель $c_{-\gamma}$ в априорной оценке

$$
\left\|u_{q}^{\varepsilon_{m}} ; W_{-\gamma}^{1,0}\left(\Pi_{+}^{\varepsilon_{m}}\right)\right\| \leqslant c_{-\gamma}\left(\left\|f_{q}^{\varepsilon_{m}} ; W_{\gamma}^{1,0}\left(\Pi_{+}^{\varepsilon_{m}}\right)^{*}\right\|+\| u_{q}^{\varepsilon_{m}} ; L_{2}\left(\mathbb{Q}_{l} \|\right)=c_{-\gamma},\right.
$$

выражающей фредгольмовость оператора $A_{-\gamma}^{\varepsilon_{m}}\left(\lambda^{\varepsilon_{m}}\right)$ (см., например, [21], теорема 5.1.4), не зависит от $\varepsilon_{m}$. Таким образом, вдоль подпоследовательности (по-прежнему обозначаем ее как $\left.\left\{\varepsilon_{m}\right\}\right)$ верны сходимости

$$
\mathbf{u}_{q}^{\varepsilon_{m}} \rightarrow \mathbf{u}_{q}^{0} \quad \text { слабо в } W_{-\gamma}^{1,0}\left(\Pi_{+}^{0}\right) \text { и сильно в } L_{2}\left(\mathbb{Q}_{l}\right),
$$

где $\mathbf{u}_{q}^{\varepsilon_{m}}$ - функции $u_{q}^{\varepsilon_{m}}$, записанные в координатах $x^{\varepsilon}$. Осуществляя предельный переход в интегральном тождестве для $\mathbf{u}_{q}^{\varepsilon_{m}}$ с фиксированной гладкой финитной пробной функцией $\mathbf{v} \in C_{c}^{\infty}\left(\Pi_{+}^{0} \cup \varpi_{0}^{0}\right)$, при помощи (85) и (81) заключаем, что

$$
\left(\nabla \mathbf{u}_{q}^{0}, \nabla \mathbf{v}\right)_{\Pi_{+}^{0}}=\pi^{2} n^{2}\left(\mathbf{u}_{q}^{0}, \mathbf{v}\right)_{\Pi_{+}^{0}}
$$

По замыканию интегральное тождество (86) переносится на все пробные функции $\mathbf{v} \in W_{\gamma}^{1,0}\left(\Pi_{+}^{0}\right)$, а значит, $\mathbf{u}_{q}^{0} \in W_{-\gamma}^{1,0}\left(\Pi_{+}^{0}\right)-$ решение однородной задачи $(30)$, причем в силу нормировки (82) имеем

$$
\left\|\mathbf{u}_{q}^{0} ; L_{2}\left(\mathbb{Q}_{l}\right)\right\|=1
$$

Принимая во внимание полный список (46) решений задачи $(86)$ в классе $W_{-\gamma}^{1,0}\left(\Pi_{+}^{0}\right)$, находим

$$
\mathbf{u}_{q}^{0}(x)=\sum_{k=1}^{n-1} c_{q}^{k} \sin \left(\pi k x_{2}\right)\left(e^{i x_{1} \pi \sqrt{n^{2}-k^{2}}}+e^{-i x_{1} \pi \sqrt{n^{2}-k^{2}}}\right)+c_{q}^{n} \sin \left(\pi n x_{2}\right) .
$$

Локальная оценка решения задачи Дирихле для оператора Лапласа

$$
\begin{gathered}
\left\|u_{q}^{\varepsilon_{m}} ; H^{2}\left(\mathbb{Q}_{l}^{\prime}\right)\right\| \leqslant c_{l}\left(\left\|f_{q}^{\varepsilon_{m}} ; L_{2}\left(\mathbb{Q}_{l}\right)\right\|+\left\|u_{q}^{\varepsilon_{m}} ; L_{2}\left(\mathbb{Q}_{l}\right)\right\|\right)=c_{l} \\
\mathbb{Q}_{l}^{\prime}=(5 l / 4,7 l / 4) \times(0,1) \subset \mathbb{Q}_{l}
\end{gathered}
$$

и компактность вложения $H^{2}\left(\mathbb{Q}_{l}\right) \subset H^{1}\left(\varpi_{l}^{0}\right)$ (см., например, [22]), где $\varpi_{l}^{\varepsilon}=\varpi_{l}^{0}-$ сечение $\left\{x: x_{1}=3 l / 2, x_{2} \in(0,1)\right\}$ волноводов, показывают, что

$$
\mathbf{u}_{q}^{\varepsilon_{m}} \rightarrow \mathbf{u}_{q}^{0} \quad \text { слабо в } H^{2}\left(\mathbb{Q}_{l}\right) \text { и сильно в } H^{1}\left(\varpi_{l}\right),
$$

может быть, вдоль подпоследовательности. На полуполосе $\Pi_{l}^{\infty}=(3 l / 2,+\infty) \times(0,1)$ применим формулу Грина к экспоненциально затухающей функции $u_{q}^{\varepsilon}$ и поочередно к ограниченным функциям

$$
\begin{aligned}
& \sin \left(\pi k x_{2}\right) e^{ \pm i\left(x_{1}-3 l / 2\right) \sqrt{\lambda_{q}^{\varepsilon} m}-\pi^{2} k^{2}}, \quad k=1, \ldots, n-1, \\
& \sin \left(\pi n x_{2}\right) e^{-\left(x_{1}-3 l / 2\right) \sqrt{\pi^{2} n^{2}-\lambda_{q}^{\varepsilon m}}},
\end{aligned}
$$


которые удовлетворяют уравнению Гельмгольца в $\Pi_{l}^{\infty}$ и условиям Дирихле на боковых сторонах полуполосы. В результате получаем набор условий ортогональности

$$
\begin{aligned}
0 & =\int_{-1}^{0} \sin \left(\pi k x_{2}\right)\left(\partial_{1} u_{q}^{\varepsilon_{m}}\left(\frac{3}{2} l, x_{2}\right) \mp i \sqrt{\lambda_{q}^{\varepsilon}-\pi^{2} k^{2}} u_{q}^{\varepsilon_{m}}\left(\frac{3}{2} l, x_{2}\right)\right) d x_{2}, \\
& k=1, \ldots, n-1, \\
0 & =\int_{-1}^{0} \sin \left(\pi n x_{2}\right)\left(\partial_{1} u_{q}^{\varepsilon_{m}}\left(\frac{3}{2} l, x_{2}\right)+\sqrt{\pi^{2} n^{2}-\lambda_{q}^{\varepsilon}} u_{q}^{\varepsilon_{m}}\left(\frac{3}{2} l, x_{2}\right)\right) d x_{2},
\end{aligned}
$$

в которых согласно (89) можно перейти к пределу при $\varepsilon_{m} \rightarrow+0$ и получить, что

$$
\begin{aligned}
0 & =\int_{-1}^{0} \sin \left(\pi k x_{2}\right)\left(\partial_{1} \mathbf{u}_{q}^{0}\left(\frac{3}{2} l, x_{2}\right) \mp i \pi \sqrt{n^{2}-k^{2}} \mathbf{u}_{q}^{0}\left(\frac{3}{2} l, x_{2}\right)\right) d x_{2}, \\
& k=1, \ldots, n-1, \\
0=\int_{-1}^{0} \sin \left(\pi n x_{2}\right) \partial_{1} \mathbf{u}_{q}^{0}\left(\frac{3}{2} l, x_{2}\right) d x_{2} . &
\end{aligned}
$$

Отсюда вытекает, что $c_{q}^{1}=\cdots=c_{q}^{n-1}=0$ в линейной комбинации (88), а условие (87) позволяет вычислить последний коэффициент $c_{q}^{n}=\sqrt{2} l^{-1 / 2}$.

Согласно утверждению 2 теоремы 4 для решений $u_{m}^{\varepsilon_{m}} \in H^{1,0}\left(\Pi_{+}^{\varepsilon_{m}}\right) \subset W_{-\gamma}^{1,0}\left(\Pi_{+}^{\varepsilon_{m}}\right)$ однородной задачи (30) верны разложения (33), в которых из-за включения в пространство Соболева многие коэффициенты обращаются в нуль:

$$
u_{q}^{\varepsilon_{m}}(x)=\widetilde{u}_{q}^{\varepsilon_{m}}(x)+b_{q}^{\varepsilon_{m}} \sin \left(\pi n x_{2}\right) e^{-x_{1} \sqrt{\pi^{2} n^{2}-\lambda_{q}^{\varepsilon}}} .
$$

При этом в вытекающей из (34) и (84) оценке

$$
\left\|\widetilde{u}_{q}^{\varepsilon_{m}} ; W_{\gamma}^{1}\left(\Pi_{+}^{\varepsilon_{m}}\right)\right\|+\left|b_{q}^{\varepsilon_{m}}\right| \leqslant c\left\|u_{q}^{\varepsilon_{m}} ; W_{-\gamma}^{1}\left(\Pi_{+}^{\varepsilon_{m}}\right)\right\| \leqslant c
$$

постоянная $c$ не зависит от малого $\varepsilon>0$ по двум причинам: во-первых, благодаря почти тождественной замене координат (38) и, во-вторых, потому что множители при $b_{q}^{\varepsilon_{m}}$ в правой части (94) имеют конечный предел $\sin \left(\pi n x_{2}\right)$ при $\varepsilon_{m} \rightarrow+0$. Следовательно, разрежая при необходимости последовательность $\left\{\varepsilon_{m}\right\}$ еще раз, получаем, что $b_{q}^{\varepsilon_{m}} \rightarrow b_{q}^{0}$ и $\widetilde{\mathbf{u}}_{q}^{\varepsilon_{m}} \rightarrow \widetilde{\mathbf{u}}_{q}^{0}$ слабо в $W_{-\gamma}^{1}\left(\Pi_{+}^{0}\right)$. Поскольку $\mathbf{u}_{q}^{0}(x)=\sqrt{2} l^{-1 / 2} \sin \left(\pi n x_{2}\right)$, обнаруживаем, что $b_{q}^{0}=\sqrt{2} l^{-1 / 2}$ и $\widetilde{\mathbf{u}}_{q}^{0}=0$. Теперь равенство (83) принимает вид

$$
0=\left(u_{1}^{\varepsilon_{m}}, u_{2}^{\varepsilon_{m}}\right)_{\Pi_{+}^{\varepsilon}}=\frac{1}{2} b_{1}^{\varepsilon_{m}} \overline{b_{2}^{\varepsilon_{m}}}\left(\left(\pi^{2} n^{2}-\lambda_{1}^{\varepsilon}\right)^{1 / 2}+\left(\pi^{2} n^{2}-\lambda_{2}^{\varepsilon}\right)^{1 / 2}\right)^{-1}+O(1) .
$$

Умножим его на бесконечно малую $\left(\pi^{2} n^{2}-\lambda_{1}^{\varepsilon}\right)^{1 / 2}+\left(\pi^{2} n^{2}-\lambda_{2}^{\varepsilon}\right)^{1 / 2}$ и перейдем к пределу вдоль подпоследовательности $\left\{\varepsilon_{m}\right\}$. В результате получаем абсурдное равенство $0=l^{-1}$, т. е. действительно соотношение (81) невозможно.

В ситуации (80) приведенные выкладки и рассуждения не претерпевают существенных изменений. В то же время функция $\mathbf{u}_{1}^{0}$ (см. (89)) оказывается решением задачи (77) с параметром $\lambda=\pi^{2}(n-1)^{2}$ и представима в виде

$$
\begin{aligned}
\mathbf{u}_{1}^{0}(x)= & \sum_{k=1}^{n-2} c_{1}^{k} \sin \left(\pi k x_{2}\right)\left(e^{i x_{1} \pi \sqrt{(n-1)^{2}-k^{2}}}+e^{-i x_{1} \pi \sqrt{(n-1)^{2}-k^{2}}}\right)+ \\
& +c_{q}^{n-1} \sin \left(\pi(n-1) x_{2}\right) .
\end{aligned}
$$


Однако, имея в распоряжении первые $n-2$ функции из списка (90), а также функцию

$$
\begin{aligned}
& \sin (\left.\pi(n-1) x_{2}\right)\left(\lambda_{1}^{\varepsilon_{m}}-\pi^{2}(n-1)^{2}\right)^{-1 / 2} \times \\
& \quad \times\left(e^{i\left(x_{1}-3 l / 2\right) \sqrt{\lambda_{1}^{\varepsilon m}-\pi^{2}(n-1)^{2}}}-e^{-i\left(x_{1}-3 l / 2\right) \sqrt{\lambda_{1}^{\varepsilon m}-\pi^{2}(n-1)^{2}}}\right)
\end{aligned}
$$

или, в случае $\lambda_{1}^{\varepsilon_{m}}=\pi^{2}(n-1)^{2}$,

$$
\left(x_{1}-\frac{3 l}{2}\right) \sin \left(\pi(n-1) x_{2}\right),
$$

получаем в дополнение к модифицированным (понятная замена $n \mapsto n-1)$ условиям ортогональности (92) еще одно условие

$$
0=\int_{-1}^{0} \sin \left(\pi(n-1) x_{2}\right) u_{1}^{0}\left(\frac{3}{2} l, x_{2}\right) d x_{2} .
$$

В итоге все коэффициенты линейной комбинации (95) обращаются в нуль, что противоречит нормировке (87), а значит, ситуация (80) действительно невозможна.

Искусственное условие Неймана появилось в задаче (6), (7) в результате предположения, что собственная функция исходной задачи (2) четная относительно переменной $x_{1}$. В случае нечетной собственной функции следует назначить на торце $\varpi_{0}^{\varepsilon}$ искусственные условия Дирихле. Решения соответствующей предельной задачи

$$
-\Delta u(x)=\lambda u(x), \quad x \in \Pi_{+}^{0}, \quad u(x)=0, \quad x \in \partial \Pi_{+}^{0},
$$

заданы формулами (78), в которых вместо знака плюс при второй экспоненте стоит знак минус. Как следствие, расширенная матрица рассеяния равна матрице (79), взятой со знаком минус. Замена $i \mapsto-i$ в формуле для $S_{n n}^{0}$ сохраняет невозможность соблюдения необходимого условия захвата волны, следовательно, число (37) не может быть собственным в случае малого $\varepsilon>0$. По прежним причинам ситуация (80) невозможна. Вместе с тем, удается отвергнуть и ситуацию $\lambda_{1}^{\varepsilon_{m}} \rightarrow \pi^{2} n^{2}-0$ (ср. соотношение (81)). Дело в том, что предыдущие рассуждения показывают, что $\mathbf{u}_{1}^{0}$ - решение задачи (96), но условия ортогональности (91) и (93) делают его нулевым, так как вместо $c_{q}^{n} \sin \left(\pi n x_{2}\right)$ в разложении (88) функции $\mathbf{u}_{1}^{0}$ фигурирует слагаемое $c_{1}^{n} x_{1} \sin \left(\pi n x_{2}\right)$. Итак, задача Дирихле в полубесконечном волноводе $\Pi_{+}^{\varepsilon}$ не имеет собственных чисел на полуинтервале $\left[\pi^{2}(n-1)^{2}, \pi^{2} n^{2}\right)$, и доказательство теоремы 1 завершено и в части единственности.

На основе проделанных расчетов можно сформулировать следующие правила: во-первых, переход собственного числа на пороговое значение сопровождается появлением у предельной задачи ограниченного решения; во-вторых, собственное число может приближаться к порогу сверху только при наличии захваченной волны у предельной задачи с пороговым значением спектрального параметра.

\section{8. АНОМАЛИИ ВУДА}

Согласно разложениям (42) с членами (52) и (54) расширенная матрица рассеяния $S^{\varepsilon}$ имеет собственные числа

$$
\sigma_{j}^{\varepsilon}=1+O(\varepsilon), \quad j=1, \ldots, n-1, \quad \sigma_{n}^{\varepsilon}=-\left(\mu+\eta_{n}^{2}\right)^{-1}\left(2 \eta_{n} \sqrt{\mu}+i\left(\eta_{n}^{2}-\mu\right)\right)+O(\varepsilon) .
$$


Пусть $\delta>0-$ малое число и

$$
\mu \in \Upsilon_{n}^{\delta}=\left(\eta_{n}^{2}-\delta, \eta_{n}^{2}+\delta\right)
$$

При $\mu=\eta_{n}^{2} \pm \delta$ справедливы неравенства $\pm \operatorname{Im} \sigma_{n}^{\varepsilon}>0$, а значит, ввиду непрерывной зависимости матрицы $S^{\varepsilon}$ от параметра (3) найдется точка $\mu_{*}^{\varepsilon} \in \Upsilon_{n}^{\delta}$, для которой $\sigma_{n}^{\varepsilon}=-1$.

Предположим, что профильная функция (10) подобрана так, что задача (6), (7) (или (2)) имеет собственное число $\lambda^{\varepsilon}=\pi^{2} n^{2}-\varepsilon^{2} \mu^{\varepsilon}$ с асимптотикой (15). Тогда $\mu_{*}^{\varepsilon}=$ $\mu^{\varepsilon}$ в силу следствия, приведенного в разделе 3 . Матрица $S^{\varepsilon}=S^{\varepsilon}(\mu)$ непрерывно зависит от $\mu$, поэтому

$$
S_{n n}^{\varepsilon}(\mu)=-1+O\left(\varepsilon+\left|\mu-\mu_{*}^{\varepsilon}\right|\right), \quad S_{\bullet n}^{\varepsilon}=O\left(\varepsilon^{1 / 2}\left(\varepsilon+\left|\mu-\mu_{*}^{\varepsilon}\right|\right)\right) .
$$

В точке $\mu=\mu_{*}^{\varepsilon}$ матрица рассеяния $s^{\varepsilon}=s^{\varepsilon}(\mu)$ совпадает с блоком $S_{\bullet \bullet}^{\varepsilon}(\mu)$, который мало отличается от единичной матрицы $\mathbb{I}_{n-1}$ размера $(n-1) \times(n-1)$, и эта близость сохраняется для точек (97), а именно,

$$
\left\|s^{\varepsilon}(\mu)-\mathbb{I}_{n-1} ; \mathbb{C}^{(n-1) \times(n-1)}\right\| \leqslant c \varepsilon .
$$

Пусть теперь нарушено хотя бы одно из равенств $(12)$, т. е. столбец $S_{\bullet n}^{\varepsilon}(\mu)$ и $S_{n \bullet}^{\varepsilon}(\mu)$ ненулевые; тогда собственное число (5) около порога $\pi^{2} n^{2}$ отсутствует, а следовательно, $S_{n n}^{\varepsilon}\left(\mu_{*}^{\varepsilon}\right) \neq-1$. Убедимся в том, что в указанных условиях у матрицы рассеяния $s^{\varepsilon}\left(\mu_{*}^{\varepsilon}\right)$ появляется собственное число -1 . Пусть $S^{\varepsilon}\left(\mu_{*}^{\varepsilon}\right) a^{\varepsilon}=-a^{\varepsilon}$ и $a^{\varepsilon}=\left(a_{\bullet}^{\varepsilon}, a_{n}^{\varepsilon}\right)-$ столбец в $\mathbb{C}^{n}$. Умножим второе уравнение системы

$$
S_{\bullet \bullet}^{\varepsilon}\left(\mu_{*}^{\varepsilon}\right) a_{\bullet}^{\varepsilon}+S_{\bullet n}^{\varepsilon}\left(\mu_{*}^{\varepsilon}\right) a_{n}^{\varepsilon}=-a_{\bullet}^{\varepsilon}, \quad S_{n \bullet}^{\varepsilon}\left(\mu_{*}^{\varepsilon}\right) a_{\bullet}^{\varepsilon}+\left(S_{n n}^{\varepsilon}\left(\mu_{*}^{\varepsilon}\right)+1\right) a_{n}^{\varepsilon}=0
$$

на $S_{\bullet n}^{\varepsilon}\left(\mu_{*}^{\varepsilon}\right)\left(S_{\bullet \bullet}^{\varepsilon}\left(\mu_{*}^{\varepsilon}\right)+1\right)^{-1}$ и вычтем результат из первого. Получаем

$$
\left(S_{\bullet \bullet}^{\varepsilon}\left(\mu_{*}^{\varepsilon}\right)-S_{\bullet n}^{\varepsilon}\left(\mu_{*}^{\varepsilon}\right)\left(S_{n n}^{\varepsilon}\left(\mu_{*}^{\varepsilon}\right)+1\right)^{-1} S_{n \bullet}^{\varepsilon}\left(\mu_{*}^{\varepsilon}\right)\right) a_{\bullet}^{\varepsilon}=-a_{\bullet}^{\varepsilon} .
$$

Равенство $a_{\bullet}^{\varepsilon}=0 \in \mathbb{C}^{n-1}$ невозможно (иначе $a_{n}^{\varepsilon} \neq 0$ и $S_{n n}^{\varepsilon}\left(\mu_{*}^{\varepsilon}\right)=-1$ в силу (99)), т. е. $a_{\bullet}^{\varepsilon}-$ собственный вектор матрицы $s^{\varepsilon}\left(\mu_{*}^{\varepsilon}\right)$, a -1 - ее собственное число.

Займемся асимптотикой матрицы рассеяния. Подставив разложения (42) в формулу (29), находим

$$
s^{\varepsilon}(\mu)=S_{\bullet \bullet}^{0}(\mu)+\varepsilon S_{\bullet \bullet}^{\prime}(\mu)-\varepsilon S_{\bullet n}^{0}(\mu)\left(1+S_{n n}^{0}(\mu)+\varepsilon S_{n n}^{\prime}(\mu)\right)^{-1} S_{n \bullet}^{0}(\mu)+\cdots .
$$

Если $\mu=\eta_{n, 0} \pm \delta \sqrt{\varepsilon}$ и число $\delta>0$ зафиксировано, то $\left|S_{n n}^{\varepsilon}(\mu)+1\right| \geqslant c \sqrt{\varepsilon}>0, c>0$ при малом $\varepsilon>0$ в силу первой из формул (52), т. е. при учете неравенств (98) и (55) мы получаем соотношение

$$
\left\|s^{\varepsilon}\left(\eta_{n, 0} \pm \delta \sqrt{\varepsilon}\right)-\mathbb{I}_{n-1} ; \mathbb{C}^{(n-1) \times(n-1)}\right\| \leqslant C \sqrt{\varepsilon} .
$$

Иными словами, все собственные числа матриц $s^{\varepsilon}\left(\eta_{n, 0} \pm \delta \sqrt{\varepsilon}\right)$ близки к единице. В то же время у той же матрицы при $\mu=\mu_{*}^{\varepsilon} \in \Upsilon_{n}^{\delta}$ появляется собственное число -1 . Таким образом, в малой окрестности точки $\mu=\eta_{n, 0}^{2}$ происходит быстрое, со скоростью $O\left(\varepsilon^{-1}\right)$, изменение матрицы рассеяния. Этот вывод можно сделать и на 
основе асимптотической формулы (100): поскольку $\mu_{*}^{\varepsilon}=\eta_{n, 0}^{2}+\varepsilon \mu^{\prime}+\cdots$ и $S_{n n}^{\varepsilon}\left(\mu_{*}^{\varepsilon}\right)=$ $-1+\varepsilon S_{n n}^{\prime}+\cdots$, поправочный член из $(100)$ превращается в $S_{\bullet n}^{0}\left(S_{n n}^{\prime}\right)^{-1} S_{n \bullet}^{0}+\varepsilon S_{\bullet \bullet}^{\prime}$ и тем самым приобретает порядок $1=\varepsilon^{0}$. Отметим, что $S_{n n}^{\prime} \neq 0$ в случае $S_{n n}^{0}=-1$, $S_{\bullet n}^{\prime} \neq 0$, так как

$1=\left|S_{n n}^{\varepsilon}\right|^{2}+\left|S_{\bullet}^{\varepsilon}\right|^{2}=\left|-1+\varepsilon S_{n n}^{\prime}+\cdots\right|^{2}+\varepsilon\left|S_{n \bullet}^{0}+\cdots\right|^{2}=1+\varepsilon\left(\left|S_{n \bullet}^{0}\right|^{2}-2 \operatorname{Re} S_{n n}^{\prime}\right)+\cdots$.

Аномально быстрые изменения дифракционной картины на околопороговых частотах были обнаружены экспериментально Вудом (см. [30]). Очень часто аномалии Вуда связывают с возникновением захваченной волны. Приведенные формулы показывают, что при наличии собственного числа (5) матрице присуща медленная изменяемость, но как раз при отсутствии собственного числа (точнее, при превращении его в точку комплексного резонанса; ср. [13]) скорость изменения некоторых элементов матрицы становится обратно пропорциональной малому параметру $\varepsilon$, описывающему возмущение (1) волновода. Максимальную скорость можно вычислить, построив при помощи приведенной в разделе 4 процедуры младшие члены разложений (42), однако в настоящей статье это не сделано.

\section{9. ОБСУЖДЕНИЕ}

Условия $H\left(x_{1}\right)=H\left(-x_{1}\right)$ и $H(0)=0$ введены в разделе 1 для упрощения изложения и сокращения вычислений. Если они нарушены, то формулы (10)-(13) заменяются следующими соотношениями:

$$
\begin{gathered}
H\left(x_{1}\right)=H_{0}\left(x_{1}\right)+\sum_{ \pm} \sum_{k=1}^{n-1} \tau_{k}^{ \pm} H_{k}^{ \pm}\left(x_{1}\right) \\
\eta_{n}\left(H_{0}\right):=2 \pi^{2} n^{2} \int_{-l}^{l} H_{0}\left(x_{1}\right) d x_{1}>0 \\
\eta_{k}^{ \pm}\left(H_{0}\right):=\frac{2 \pi n k}{\sqrt{n^{2}-k^{2}}} \int_{-l}^{l} H_{0}\left(x_{1}\right) e^{x_{1} \pi \sqrt{n^{2}-k^{2}}} d x_{1}=0 \\
\eta_{k}^{\alpha}\left(H_{j}^{\beta}\right)=\delta_{k, j} \delta_{\alpha, \beta}, \quad k, j=1, \ldots, n-1, \quad \alpha, \beta= \pm .
\end{gathered}
$$

Таким образом, увеличивается количество дополнительных параметров, образующих вектор $\vec{\tau}=\left(\tau_{0}, \tau_{1}^{ \pm}, \ldots, \tau_{n-1}^{ \pm}\right)$и определяемых при решении нелинейного уравнения вида $(76)$ в $\mathbb{R}^{2 n-1}$. Матрицы рассеяния, классическая и расширенная, также увеличивают свои размеры до $2(n-1) \times 2(n-1)$ и $2 n \times 2 n$ соответственно. Тем не менее все выкладки и рассуждения в целом сохраняются и позволяют сформулировать следующую теорему.

ТЕОРЕМа 8. Пусть $n>1$ и выполнены условия (101). Найдутся такие положительные $\varepsilon_{0}$ u $K$, что при $\varepsilon \in\left(0, \varepsilon_{0}\right]$ существуют параметры $\tau_{j}^{ \pm} \in(-K \varepsilon, K \varepsilon), j=$ $1, \ldots, n-1$, при которых у задачи $(2)$ в волноводе $\Pi^{\varepsilon}$ на интервале $\left(\pi^{2}(n-1)^{2}, \pi^{2} n^{2}\right)$ имеется единственное собственное число и справедливо соотношение

$$
\left|\lambda^{\varepsilon}-\pi^{2} n^{2}-\varepsilon^{2} \pi^{4} n^{4}\left(\int_{-l}^{l} H\left(x_{1}\right) d x_{1}\right)^{2}\right| \leqslant K \varepsilon^{3} .
$$


Возможны и иные простые, не требующие новых соображений, обобщения постановки задачи, например возмущение обеих сторон полосы $\Pi^{0}$ или кусочно-гладкие профильные функции $H$.

Результат работы [6] и теорем 1,8 настоящей работы показывают, что точечный спектр задачи (2) может содержать два собственных числа, одно в дискретном, а другое в непрерывном спектре. Вопрос о построении локального возмущения квантового волновода, порождающего большее количество собственных чисел, остается открытым. Ввиду специфики построения нелинейного уравнения этот вопрос не может быть решен на основе разработанного подхода.

Благодарности. Работа выполнена при финансовой поддержке РФФИ (грант № 09-01-00759).

\section{Список литературы}

[1] М.Ш. Бирман, М. З. Соломяк, Спектральная теория самосопряженных операторов в гильбертовом пространстве, Учеб. пособие, Из-во ЛГУ, Л., 1980.

[2] В. П. Маслов, Докл. АН СССР, 123:4 (1958), 631-633.

[3] В. П. Маслов, Е. М. Воробьев, Докл. АН СССР, 179:3 (1968), 558-561.

[4] P. Exner, P. Šeba, J. Math. Phys., 30:11 (1989), 2574-2580.

[5] P. Duclos, P. Exner, Rev. Math. Phys., 7:1 (1995), 73-102.

[6] W. Bulla, F. Gesztesy, W. Renger, B. Simon, Proc. Amer. Math. Soc., 125:5 (1997), 1487-1495.

[7] P. Exner, S. A. Vugalter, Lett. Math. Phys., 39:1 (1997), 59-68.

[8] D. Borisov, P. Exner, R. Gadyl'shin, D. Krejčiřik, Ann. Henri Poincaré, 2:3 (2001), 553-572, arXiv: math-ph/0011052.

[9] В. В. Грушин, Матем. заметки, 75:3 (2004), 360-371.

[10] Р. Р. Гадыльшин, ТМФ, 145:3 (2005), 358-371.

[11] С. А. Назаров, Сиб. матем. журн., 51:5 (2010), 1086-1101.

[12] D. V. Evans, M. Levitin, D. Vassiliev, J. Fluid Mech., 261 (1994), 21-31.

[13] A. Aslanyan, L. Parnovski, D. Vassiliev, Quart. J. Mech. Appl. Math., 53:3 (2000), 429-447.

[14] И. В. Камоцкий, С. А. Назаров, Матем. сб., 190:1 (1999), 109-138.

[15] И. В. Камоцкий, С. А. Назаров, Матем. сб., 190:2 (1999), 43-70.

[16] С. А. Назаров, Б. А. Пламеневский, Алгебра и анализ, 6:4 (1994), 157-186.

[17] И. В. Камоцкий, С. А. Назаров, "Расширенная матрица рассеяния и экспоненциально затухающие решения эллиптической задачи в цилиндрической области", Maтематические вопросы теории распространения волн, т. 29, Зап. научн. сем. ПОМИ, 264, ред. В. М. Бабич, ПОМИ, СПб., 2000, 66-82.

[18] С. А. Назаров, УМH, 54:5(329) (1999), 77-142.

[19] С. А. Назаров, Функи. анализ и его прил., 40:2 (2006), 20-32.

[20] В. А. Кондратьев, "Краевые задачи для эллиптических уравнений в областях с коническими или угловыми точками", Тр. ММО, 16, Изд-во Моск. ун-та, М., 1967, 209-292.

[21] С. А. Назаров, Б. А. Пламеневский, Эллиптические задачи в областях с кусочно гладкой гранищей, Наука, М., 1991.

[22] О.А. Ладыженская, Краевые задачи математической физики, Наука, М., 1973.

[23] В. Г. Мазья, Б. А. Пламеневский, Math. Nachr., 76:1 (1977), 29-60.

[24] В. Г. Мазья, Б. А. Пламеневский, Math. Nachr., 81:1 (1978), 25-82.

[25] S. A. Nazarov, "Properties of spectra of boundary value problems in cylindrical and quasicylindrical domains", Sobolev Spaces in Mathematics, v. II, Int. Math. Ser. (N.Y.), 9, ed. V. Maz'ya, Springer, New York, 2009, 261-309. 
[26] А.М. Ильин, Согласование асимптотических разложений решений краевых задач, Наука, М., 1989.

[27] В. И. Смирнов, Курс высшей математики, т. 2, Наука, М., 1967.

[28] С. А. Назаров, Б. А. Пламеневский, "Принципы излучения для самосопряженных эллиптических задач", Дифференииальные уравнения. Спектральная теория. Теория распространения волн, Проблемы матем. физики, 13, ред. М. Ш. Бирман, Изд-во ЛГУ, Л., 1991, 192-244.

[29] W. G. Mazja, S. A. Nazarov, B. A. Plamenewski, Asymptotische Theorie elliptischer Randwertaufgaben in singulär gestörten Gebieten, v. 1, Math. Lehrbucher Monogr., 82, Akademie, Berlin, 1991.

[30] В. Сибрук, Роберт Вилъямс Вуд. Современный чародей физической лаборатории, Гос. изд. технико-теоретической лит-ры, М., Л., 1946.

Поступила в редакцию 12.11.2010 